\title{
Reasoning on Divergent Computations with Coaxioms
}

\author{
DAVIDE ANCONA, Università di Genova, Italy \\ FRANCESCO DAGNINO, Università di Genova, Italy \\ ELENA ZUCCA, Università di Genova, Italy
}

Coaxioms have been recently introduced to enhance the expressive power of inference systems, by supporting interpretations which are neither purely inductive, nor coinductive. This paper proposes a novel approach based on coaxioms to capture divergence in semantic definitions by allowing inductive and coinductive semantic rules to be merged together for defining a unique semantic judgment. In particular, coinduction is used to derive a special result which models divergence. In this way, divergent, terminating, and stuck computations can be properly distinguished even in semantic definitions where this is typically difficult, as in big-step style. We show how the proposed approach can be applied to several languages; in particular, we first illustrate it on the paradigmatic example of the $\lambda$-calculus, then show how it can be adopted for defining the big-step semantics of a simple imperative Java-like language. We provide proof techniques to show classical results, including equivalence with small-step semantics, and type soundness for typed versions of both languages.

\section{CCS Concepts: • Theory of computation $\rightarrow$ Operational semantics;}

Additional Key Words and Phrases: Big-step operational sematics, inductive and coinductive inference systems, type soundness

\section{ACM Reference Format:}

Davide Ancona, Francesco Dagnino, and Elena Zucca. 2017. Reasoning on Divergent Computations with Coaxioms. Proc. ACM Program. Lang. 1, OOPSLA, Article 81 (October 2017), 26 pages. https://doi.org/10.1145/ 3133905

\section{INTRODUCTION}

An important issue in semantic definitions of programming languages is to properly model and allow reasoning on non termination. Let us consider, for instance, small-step and big-step operational semantics. Both are formally expressed as relations (judgments) defined by an inference system, so that each judgment has a finite proof tree. However, in small-step semantics the judgment models a single step of computation, hence a divergent computation is modeled as an infinite sequence of steps. This has the advantage that non terminating computations are clearly distinguished from stuck computations, and for this reason small-step semantics is usually preferred to prove soundness of type systems by progress and subject reduction properties, as pioneered by Wright and Felleisen [Wright and Felleisen 1994]. However, in this way divergence is modeled at the meta-level rather than by an inference system. On the other side, in big-step semantics the judgment models the

Authors' addresses: DIBRIS, Università di Genova, via Dodecaneso 35, 16146, Genova, Italy

Authors' email addresses:\{davide. ancona, elena.zucca\}@unige.it,fra.dagn@gmail.com.

Authors' addresses: Davide Ancona, DIBRIS, Università di Genova, Italy, davide.ancona@unige.it; Francesco Dagnino, DIBRIS, Università di Genova, Italy, fra.dagn@gmail.com; Elena Zucca, DIBRIS, Università di Genova, Italy, elena.zucca@ unige.it.

Permission to make digital or hard copies of all or part of this work for personal or classroom use is granted without fee provided that copies are not made or distributed for profit or commercial advantage and that copies bear this notice and the full citation on the first page. Copyrights for components of this work owned by others than the author(s) must be honored Abstracting with credit is permitted. To copy otherwise, or republish, to post on servers or to redistribute to lists, requires prior specific permission and/or a fee. Request permissions from permissions@acm.org.

(C) 2017 Copyright held by the owner/author(s). Publication rights licensed to Association for Computing Machinery. 2475-1421/2017/10-ART81

https://doi.org/10.1145/3133905

Proceedings of the ACM on Programming Languages, Vol. 1, No. OOPSLA, Article 81. Publication date: October 2017. 
overall result of a computation, hence non terminating and stuck computations are identified, since in both cases there exist no derivable judgment.

In both approaches, it is possible to express divergence as well by an inference system, by introducing a separate judgment defined by inference rules which are interpreted coinductively [Cousot and Cousot 1992; Leroy and Grall 2009]. Leroy and Grall [Leroy and Grall 2009] investigate also the possibility of providing a unique definition by interpreting coinductively the standard rules for the big-step operational semantics (coevaluation). In other words, showing that a computation returns a result by a possibly infinite proof tree. Unfortunately, this approach does not fully capture divergence. Indeed, first of all coevaluation is non deterministic, in the sense that some diverging terms, e.g., $\Omega=(\lambda x . x x)(\lambda x . x x)$, coevaluate to any value. On the other hand, with a left-to-right call-by-value evaluation strategy some other diverging terms, e.g., $\Omega(00)$, do not coevaluate as expected, that is, still have no proof tree. See Sect. 3 at page 8 for a formal account.

Another drawback of coevaluation is that there exist well-typed non terminating terms for which the coinductive semantics is not defined, since only well-founded values are considered. In [Ancona 2012] it is shown that, for a Java-like language, by considering non well-founded values as well, the claim of soundness holds when expressed in terms of a coinductive big-step semantics. Unfortunately, the proof requires advanced mathematical concepts based on the notion of complete or compact metric space.

Coaxioms are a recently introduced notion [Ancona et al. 2017] which enhances the expressivity of inference systems, by supporting interpretations which are neither purely inductive, nor coinductive. This paper proposes a novel approach based on coaxioms to capture divergence in semantic definitions by allowing inductive and coinductive semantic rules to be merged together for defining a unique semantic judgment. In particular, coinduction is used to derive a special result which models divergence. In this way, divergent, terminating, and stuck computations can be properly distinguished even in semantic definitions where this is typically difficult, as we demonstrate for the big-step style. This semantics can be seen as a filtered coevaluation. Indeed, it is coevaluation in the sense that infinite proof trees are allowed, but appropriate coaxioms are introduced to filter out unwanted results. Notably, for all terms expected to diverge, it is only possible to derive divergence as result, and no spurious values can be obtained. This solves all the above mentioned problems of coevaluation.

We show how proofs can be performed in this framework, notably equivalence with small-step semantics and type soundness. We first illustrate the approach on the paradigmatic example of the $\lambda$-calculus, then show its effectiveness on a simple imperative Java-like language.

The paper is structured as follows. Sect. 2 briefly recalls the notions and results from [Ancona et al. 2017] needed to make the paper self-contained. In Sect. 3 we introduce the approach using the $\lambda$-calculus as example. Then, we consider a simple imperative Java-like language in Sect. 4. Sect. 5 contains the complete proof of type soundness. Finally, the most relevant related work is surveyed in Sect. 6, and Sect. 7 concludes and outlines directions for further investigation.

\section{COAXIOMS IN A NUTSHELL}

In this section, we provide a short introduction to generalized inference systems [Ancona et al. 2017]. Although two equivalent model- and proof-theoretic semantics are provided for generalized inference systems [Ancona et al. 2017], we focus here on the more intuitive proof-theoretic view, which is crucial in this paper to understand how coaxioms can be used to model divergence.

First of all we recall standard notions about inference systems [Aczel 1977; Leroy and Grall 2009]. Assume a set $\mathcal{U}$ called the universe, whose elements are called judgments. An inference system consists of a set of inference rules, which are pairs $\frac{\operatorname{Pr}}{c}$, with $\operatorname{Pr} \subseteq \mathcal{U}$ the set of premises, $c \in \mathcal{U}$ the 
consequence (a.k.a. conclusion). A rule with an empty set of premises is called an axiom. A proof tree is a tree whose nodes are (labeled with) judgments in $\mathcal{U}$, and there is a node $c$ with set of children $\operatorname{Pr}$ only if there exists a rule $\frac{P r}{c}$.

The inductive interpretation of $\mathcal{I}$, denoted $\operatorname{Ind}(\mathcal{I})$, is the set of judgments which are the root of a finite ${ }^{1}$ proof tree, whereas the coinductive interpretation of $\mathcal{I}$, denoted $\operatorname{CoInd}(\mathcal{I})$, is the set of judgments which are the root of an arbitrary (finite or infinite) proof tree.

Both interpretations can also be characterized set-theoretically as follows. We define the (one step) inference operator $F_{\mathcal{I}}: \wp(\mathcal{U}) \rightarrow \wp(\mathcal{U})$ by $F_{\mathcal{I}}(S)=\left\{c \mid \operatorname{Pr} \subseteq S, \frac{\operatorname{Pr}}{c} \in \mathcal{I}\right\}$. A set $S$ is closed if $F_{\mathcal{I}}(S) \subseteq S$, and consistent if $S \subseteq F_{\mathcal{I}}(S)$. That is, no new judgments can be inferred from a closed set, and all judgments in a consistent set can be inferred from the set itself. Then, it can be proved that $\operatorname{Ind}(\mathcal{I})$ is the smallest closed set, that is, the intersection of all closed sets, and $\operatorname{CoInd}(\mathcal{I})$ is the largest consistent set, that is, the union of all consistent sets.

We describe now the notion of coaxiom and generalized inference system [Ancona et al. 2017].

Definition 2.1 (Generalized inference system). A generalized inference system, or inference system with coaxioms, is a pair $(\mathcal{I}, \gamma)$ consisting of an inference system $\mathcal{I}$ and a set of coaxioms $\gamma$, with $\gamma \subseteq \mathcal{U}$.

A coaxiom $c$ is written $\frac{\bullet}{c}$, very much like an axiom, and analogously to an axiom it can be used as an initial assumption to derive other judgments. However, coaxioms are used in a special way, explained in the following.

Let us consider an introductory example which computes the judgment $n \stackrel{\star}{\rightarrow} \mathcal{N}$ meaning that $\mathcal{N}$ is the set of nodes reachable from a node $n$ of a given graph. Let us represent a graph by its set of nodes $V$ and a function adj which returns all the adjacents of a node. As usual, sets of rules can be expressed by a metarule with side conditions, and analogously sets of coaxioms can be expressed by a metacoaxiom with side conditions.

$$
\frac{n_{1} \stackrel{\star}{\rightarrow} \mathcal{N}_{1} \ldots n_{k} \stackrel{\star}{\rightarrow} \mathcal{N}_{k}}{n \stackrel{\star}{\rightarrow}\{n\} \cup \mathcal{N}_{1} \cup \ldots \cup \mathcal{N}_{k}} \operatorname{adj}(n)=\left\{n_{1}, \ldots, n_{k}\right\} \quad \stackrel{\bullet}{\stackrel{\bullet}{\rightarrow} \emptyset} n \in V
$$

For instance, in the case of a graph with nodes $a, b, c$, with an arc from $a$ into $b$ and conversely, and $c$ isolated, we would get the following metarules and coaxioms:

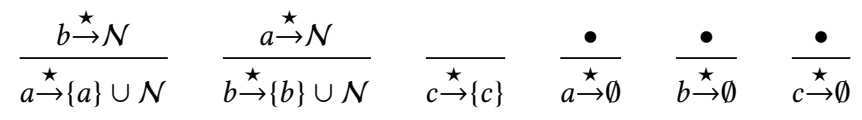

To show the aim of coaxioms, let us first consider what happens if we interpret the metarules either inductively or coinductively, disregarding the coaxioms. If we interpret the metarules inductively (excluding the coaxioms), then we get only the judgment $c \stackrel{\star}{\rightarrow}\{c\}$. In other words, a visit computing $n \stackrel{\star}{\rightarrow} \mathcal{N}$, like other judgments on graphs, should mark already encountered nodes to avoid non termination, since the graph structure is not well-founded. ${ }^{2}$

On the other hand, if we interpret the metarules coinductively (excluding again the coaxioms), then we get the correct judgments $a^{\star} \stackrel{\star}{\rightarrow}\{a, b\}$ and $b \stackrel{\star}{\rightarrow}\{a, b\}$, but we also get the wrong judgments

\footnotetext{
${ }^{1}$ Under the common assumption that sets of premises are finite, otherwise we should say a well-founded tree, that is, a tree with no infinite paths.

${ }^{2}$ Note that, instead, the judgment $n \stackrel{\star}{\rightarrow} n^{\prime}$, meaning that $n^{\prime}$ is reachable from $n$, can be trivially defined inductively. However, there is no simple way to define by an inference system $n \stackrel{\star}{\rightarrow} \mathcal{N}$, with $\mathcal{N}$ all nodes reachable from $n$, in terms of $n \stackrel{\star}{\rightarrow} n^{\prime}$.
} 
$a \stackrel{\star}{\rightarrow}\{a, b, c\}$ and $b \stackrel{\star}{\rightarrow}\{a, b, c\}$. For instance, the judgment $\stackrel{\star}{\rightarrow}\{a, b, c\}$ has the infinite proof tree shown below.

$$
\frac{\frac{\vdots}{a \stackrel{\star}{\rightarrow}\{a, b, c\}}}{\frac{b^{\star}\{a, b, c\}}{a \star}\{a, b, c\}}
$$

Coaxioms come into play when we consider a different interpretation, called interpretation generated by coaxioms [Ancona et al. 2017] and denoted $\operatorname{Gen}(\mathcal{I}, \gamma)$, defined below. Let $\mathcal{I}_{\sqcup \gamma}$ denote the inference system obtained by enriching $\mathcal{I}$ with judgments in $\gamma$ considered as axioms.

Definition 2.2 (Interpretation generated by coaxioms). Let $(\mathcal{I}, \gamma)$ be a generalized inference system. Then, the interpretation generated by coaxioms $\operatorname{Gen}(\mathcal{I}, \gamma)$ is the set of judgments which have an arbitrary (finite or infinite) proof tree in $\mathcal{I}$, whose nodes all have a finite proof tree in $\mathcal{I}_{\sqcup \gamma}$.

Note that, given an arbitrary proof tree in $\mathcal{I}$, its nodes which are roots of a finite subtree always satisfy the condition required by the definition above (a finite proof tree in $\mathcal{I}$ is a finite proof tree in $\mathcal{I}_{\sqcup \gamma}$ as well), hence the condition is only significant for nodes which are roots of an infinite path in the proof tree.

For instance, in the example, the judgment $a \stackrel{\star}{\rightarrow}\{a, b\}$ has an infinite proof tree in $\mathcal{I}$ where each node has a finite proof tree in $\mathcal{I}_{\sqcup \gamma}$, as shown below.

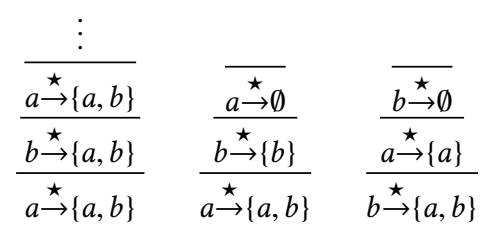

As the example shows, coaxioms filter out undesired infinite proof trees.

Note that the inductive and coinductive interpretation can be obtained as special cases of the interpretation generated by coaxioms of an inference system, notably:

- the inductive interpretation when the set of coaxioms is empty

- the coinductive interpretation when the set of coaxioms is the universe.

In set-theoretic terms, $\operatorname{Gen}(\mathcal{I}, \gamma)=\operatorname{CoInd}\left(\mathcal{I}_{\sqcap \operatorname{Ind}\left(\mathcal{I}_{\amalg_{\gamma}}\right)}\right)$, that is:

(1) first, we take the inductive interpretation of $\mathcal{I}_{\sqcup \gamma}$

(2) then, we take the coinductive interpretation of the inference system $\mathcal{I}_{\sqcap \operatorname{Ind}\left(\mathcal{I}_{{ }_{Y}}\right)}$ obtained from

$\mathcal{I}$ by keeping only rules with consequence in $\operatorname{Ind}\left(\mathcal{I}_{\sqcup \gamma}\right)$.

In [Ancona et al. 2017] it is shown that this corresponds to taking a fixed point of $F_{\mathcal{I}}$ which is, in general, neither the least, nor the greatest, called bounded fixed-point generated by $\gamma$.

We show now how some recursive definitions of functions can be expressed as inference systems with coaxioms. Let $\mathbb{Z}$ denote the set of integers, $\mathbb{L}$ the set of (finite and infinite) lists of integers, $\Lambda$ the empty list and $x: l$ the list with head $x$ and tail $l$.

The first example is the function which checks whether all the elements of a list are positive, expressed by judgments of shape allPos $(l, b)$ with $l \in \mathbb{L}$ and $b \in\{T, F\}$.

$$
\overline{\operatorname{allPos}(\Lambda, T)} \quad \frac{\operatorname{allPos}(l, b)}{\operatorname{allPos}(x: l, F)} x \leq 0 \quad \frac{\bullet}{\operatorname{allPos}(x: l, b)} x>0 \quad \frac{\bullet}{\operatorname{allPos}(l, T)}
$$


With the coaxioms, we obtain the expected function also on infinite lists of positives: indeed, we only consider the infinite trees where the nodes have a finite proof tree in the inference system enriched by the coaxioms. In this way, the infinite tree where $b=F$ is filtered out.

The function which checks whether an element belongs to a list, expressed by judgments of shape member $(x, l, b)$ with $x \in \mathbb{Z}, l \in \mathbb{L}$ and $b \in\{T, F\}$, is a very similar example, with the difference that the coaxioms map every list into false rather than true. The only aim of the side condition in the third rule is avoiding useless rule overlapping.

$$
\overline{\operatorname{member}(x, \Lambda, F)} \quad \frac{\operatorname{member}(x, l, b)}{\operatorname{member}(x, x: l, T)} \quad \frac{\bullet}{\operatorname{member}(x, y: l, b)} x \neq y \quad \frac{\bullet}{\operatorname{member}(x, l, F)}
$$

Analogously to the previous example, with the coaxioms we obtain the expected result also on infinite lists which do not contain the element.

The function which returns the set of the elements contained in a list (with finite carrier) is expressed by judgments of shape elems $(l, x s)$, with $l \in \mathbb{L}$ and $x s \in \wp_{f}(\mathbb{Z})$, where elements of $\wp_{f}(\mathbb{Z})$ are finite sets of integers. ${ }^{3}$

$$
\frac{\operatorname{elems}(l, x s)}{\operatorname{elems}(\Lambda, \emptyset)} \quad \frac{\bullet}{\operatorname{elems}(x: l,\{x\} \cup x s)} \quad \frac{\bullet}{\operatorname{elems}(l, \emptyset)}
$$

In this case, without coaxioms the inductive interpretation gives the expected result only on finite lists, and the coinductive interpretation fails to be a function on infinite lists. For instance, for $l$ the infinite list of $1 \mathrm{~s}$, any judgment elems $(l, x s)$ with $1 \in x s$ can be derived. Indeed, for any such judgment we can construct an infinite proof tree which is a chain of applications of the last metarule. With the coaxioms, we only consider the infinite trees where the node elems $(l, x s)$ has a finite proof tree in the inference system enriched by the coaxioms. This is only true for $x s=\{1\}$.

Finally, the function which returns the greatest element contained in a (non empty) list is expressed by judgments of shape $\max (l, x)$, with $l \in \mathbb{L}$ and $x \in \mathbb{Z}$.

$$
\overline{\max (x: \Lambda, x)} \quad \frac{\max (l, y)}{\max (x: l, z)} z=\max (x, y) \quad \frac{\bullet}{\max (x: l, x)}
$$

Analogously to the previous example, without coaxioms the coinductive interpretation fails to be a function (for instance, for $l$ the infinite list of 1 s, any judgment $\max (l, x)$ with $x \geq 1$ can be derived), and the coaxioms "filter out" the wrong results.

Several proof techniques have been proposed for inference systems with coaxioms [Ancona et al. 2017]. For the aim of this paper, we only need the bounded coinduction principle illustrated below, which is a generalization of the standard coinduction principle.

Assume that $\operatorname{Gen}(\mathcal{I}, \gamma)$ is the interpretation generated by coaxioms for some $(\mathcal{I}, \gamma)$, and that $\mathcal{S}$ (for "specification") is the intended set of judgments, called valid in the following.

Completeness, that is, the property that each valid judgment can be derived $(\mathcal{S} \subseteq \operatorname{Gen}(\mathcal{I}, \gamma))$, can be proved as follows:

THEOREM 2.3 (Bounded COINDUCTION PRINCIPLE). If the following two conditions hold:

(1) $\mathcal{S} \subseteq \operatorname{Ind}\left(\mathcal{I}_{\sqcup \gamma}\right)$, that is, each valid judgment has a finite proof tree in $\mathcal{I}_{\sqcup \gamma}$;

(2) $\mathcal{S} \subseteq F_{\mathcal{I}}(\mathcal{S})$, that is, each valid judgment is the consequence of an inference rule in $\mathcal{I}$ where all premises are in $\mathcal{S}$

then $\mathcal{S} \subseteq \operatorname{Gen}(\mathcal{I}, \gamma)$.

\footnotetext{
${ }^{3} \mathrm{~A}$ more complex definition can be provided to handle lists with infinite carrier as well. However, here our aim is to show how the obvious recursive definition for finite lists works also for regular (cyclic) lists by using coaxioms.
} 
The standard coinduction principle can be obtained as a specific instance of the principle above, when $\gamma=\mathcal{U}$; for this particular case the first condition trivially holds.

We illustrate the technique on the inference system with coaxioms $(\mathcal{I}, \gamma)$ which defines the judgment allPos $(l, b)$ (page 4 ). Set $\mathcal{S}^{\text {allPos }}$ the set of pairs $(l, b)$ where $b$ is $T$ if all the elements in $l$ are positive, $F$ otherwise. Completeness means that the judgment allPos $(l, b)$ can be proved for all $(l, b) \in \mathcal{S}^{\text {allPos }}$. By the bounded coinduction principle, we have to prove conditions (1) and (2). To prove the first condition, we have to show that, for each $(l, b) \in \mathcal{S}^{\text {allPos }}$, allPos $(l, b)$ has a finite proof tree in $\mathcal{I}_{\sqcup \gamma}$. This can be easily shown, indeed:

- If $l$ contains non positive elements, let us denote with $x$ the first of such elements, hence $l=x_{1}: \ldots: x_{n}: x: l^{\prime}$ with $x_{i}>0$ for $i \in[1 . . n], x \leq 0$, and $b=F$

then we can reason by arithmetic induction on $n$. Indeed, for $n=0,(l, b)$ is the consequence of the second rule with no premises, and for $n>0$ it is the consequence of the third rule where we can apply the inductive hypothesis to the premise.

- If $l$ contains only positive elements, hence $b=T$, then $(l, b)$ is a coaxiom, hence it is the consequence of a rule with no premises in $\mathcal{I}_{\sqcup \gamma}$.

To prove the second condition, we have to show that, for each $(l, b) \in \mathcal{S}^{\text {allPos }}$, allPos $(l, b)$ is the consequence of a rule with premises in $\mathcal{S}^{\text {allPos }}$. This can be easily shown, indeed:

- If $l=\Lambda$, hence $b=T$, then $\operatorname{allPos}(\Lambda, T)$ is the consequence of the first rule with no premises.

- If $l=x: l^{\prime}$ with $x \leq 0$, hence $b=F$, then $\operatorname{allPos}(l, F)$ is the consequence of the second rule with no premises.

- If $l=x: l^{\prime}$ with $x>0$, and $b=T$, hence $\left(l^{\prime}, T\right) \in \mathcal{S}^{\text {allPos }}$, then allPos $(l, T)$ is the consequence of the third rule with premise $\left(l^{\prime}, T\right)$, and analogously if $b=F$.

To show that the inference system with coaxioms exactly defines the valid judgments, we also need to prove soundness, that is, that allPos $(l, b)$ can only be proved if $(l, b) \in \mathcal{S}^{\text {allPos }}$. Such soundness proof can be found in [Ancona et al. 2017].

\section{COAXIOMS FOR DIVERGENCE}

In this section we show how coaxioms can be used to provide a big-step semantics which includes divergence among the possible results of a computation.

In the following, given an inference system with coaxioms $(\mathcal{I}, \gamma)$, we will often simply write a judgment $j$ to mean that $j$ is derivable in $(\mathcal{I}, \gamma)$, as it is common practice. However, when this is needed to avoid confusion, we will explicitly write $(\mathcal{I}, \gamma) \vdash j$, and we will abbreviate $(\mathcal{I}, \emptyset) \vdash j$ by $\mathcal{I} \vdash j$.

We assume a universe $\left\{c \Rightarrow r_{\infty} \mid c \in C, r_{\infty} \in R \cup\{\infty\}\right\}$ of big-step judgments, where $C$ and $R$ are sets of configurations and (standard) results, respectively, with $\infty \notin R$. The big-step judgment has general shape $c \Rightarrow r_{\infty}$, meaning that either $c$ evaluates to a standard result, when $r_{\infty} \neq \infty$, or diverges, when $r_{\infty}=\infty$.

Big-step semantics will be expressed by a generalized inference system $(\mathcal{I}, \gamma)$, with $\gamma=\{c \Rightarrow$ $\infty \mid c \in C\}$, which satisfies the following conservativity property.

Definition 3.1. $(\mathcal{I}, \gamma)$ is conservative if $(\mathcal{I}, \gamma) \vdash c \Rightarrow r_{\infty}$ implies that $r_{\infty} \neq \infty$ if and only if $\mathcal{I} \vdash c \Rightarrow r_{\infty}$.

Informally, this means that including divergence among the results of a computation should not affect the result of non divergent computations. More precisely, the definition above corresponds to require that:

- non divergent computations are always derivable without using the coaxioms, that is, with a finite proof tree 
- divergent computations are only derivable by using the coaxioms, that is, with an infinite proof tree.

We illustrate the notion by the standard call-by-value $\lambda$-calculus. In this simple case, configurations and results correspond to expressions and values of the calculus, respectively. In more complex languages, configurations and results will typically include auxiliary structures, as will be shown in Sect. 4.

Fig. 1 shows syntax and big-step semantics as initially defined by Ancona et al. [Ancona et al. 2017]. In this section, besides showing an initial simple example of coevaluation with coaxioms, our novel contribution is formally proving several expected properties of the semantic definition: conservativity w.r.t. the standard inductive big-step semantics, equivalence with the small-step semantics, and soundness of simply-typed $\lambda$-calculus with recursive types. We assume infinite sets

$$
\begin{aligned}
& e::=v|x| e_{1} e_{2} \text { expression } \\
& v::=i \mid \lambda x . e \quad \text { value } \\
& v_{\infty}::=v \mid \infty \quad \text { result }
\end{aligned}
$$

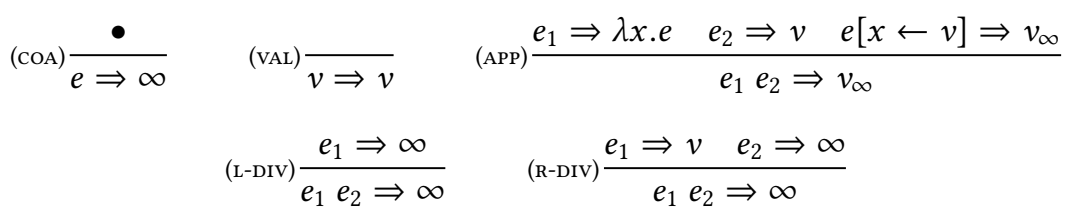

Fig. 1. $\lambda$-calculus: syntax and big-step semantics

of variables $x$ and integer constants $i$.

The unique coaxiom ${ }^{4}$ ensures that an infinite proof tree is only allowed for deriving divergence. Rule (VAL) is standard. Rule (APP) looks the standard application rule. However, note that $v_{\infty}$ is used for the result, that is, the rule also covers the case when the evaluation of the body of the lambda abstraction diverges. On the other hand, application is performed only when both expressions $e_{1}$ and $e_{2}$ do not diverge, as indicated by the meta-variable $v$ in the second premise. As usual, $e[x \leftarrow v]$ denotes capture-avoiding substitution modulo $\alpha$-renaming. Rules (L-DIv) and (R-DIv) cover the cases when either $e_{1}$ or $e_{2}$ diverges when trying to evaluate application, assuming that a left-to-right evaluation strategy has been imposed.

As an example, we show that the only judgment derivable for $\Omega=(\lambda x . x x)(\lambda x \cdot x x)$ is $\Omega \Rightarrow \infty$. Indeed, we can exhibit an infinite proof tree, not using the coaxiom, for the judgment $\Omega \Rightarrow v_{\infty}$, for all $v_{\infty}$ :

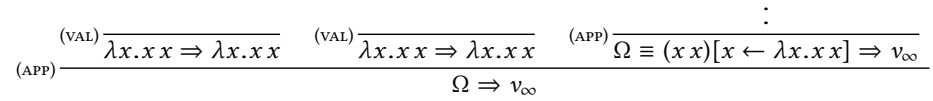

In this particular case the proof tree is also regular, but of course there are examples of divergent computations whose proof tree is not regular. The vertical dots indicate that the proof continues with the same repeated pattern.

Then, we have to construct a finite proof tree, employing also the coaxiom, for all the nodes of the above infinite proof tree. It is easy to see that this is only possible when $v_{\infty}=\infty$. In other

\footnotetext{
${ }^{4}$ Here and in what follows, as it is common practice, we will often write "rule" and "coaxiom" meaning metarule and metacoaxiom.
} 
words, the coaxiom plays a crucial role by filtering out all undesired results, leaving only the result $\infty$ representing divergence.

Coevaluation as in [Ancona 2012; Leroy and Grall 2009] corresponds to considering only rules (VAL) and (APP) of Fig. 1 and interpreting them coinductively. Then, as mentioned in the Introduction, the term $\Omega$ coevaluates to any value, by the infinite derivation shown above. However, $\Omega(00)$ has no derivation since the subterm 00 has no derivation: there exists no $v_{\infty}$ such that 00 coevaluates to $v_{\infty}$. In other words, a non terminating term applied to a "wrong" term has no derivation, whereas it should be non terminating (assuming left-to-right evaluation). In our approach, instead, for the term $\Omega(00)$ we correctly derive the judgment $\Omega(00) \Rightarrow \infty$, by rule (L-Div).

It is easy to see that a judgment of shape $e \Rightarrow v$ is derivable if and only if it corresponds to the usual big-step semantics of $\lambda$-calculus, that is, the judgment inductively defined by the inference system which only includes rules (VAL) and (APP) (note, indeed, that rules (L-Div) and (R-DIv) are useless without the coaxiom). In other words, values obtained as results are exactly those obtained by the inference system which does not consider divergence. This is formally expressed by the theorem below.

Theorem 3.2 (Conservativity). If $e \Rightarrow v_{\infty}$ holds, then $v_{\infty} \neq \infty$ if and only if the judgment has a finite proof tree.

For the proof we refer to the analogous proof of conservativity (Theorem 4.1) for the imperative Java-like language in Sect. 4.

Given a conservative big-step semantics, we will often need to prove completeness of divergence with respect to a given subset $C$ of configurations (corresponding to a property of interest), that is, that all the configurations in $C$ which do not reduce to a standard result should diverge. ${ }^{5}$

This can be proved with an instantiation of the bounded coinduction principle.

Theorem 3.3 (Divergence Consistency Principle). Let $(\mathcal{I}, \gamma)$ be a conservative big-step semantics. Given $C \subseteq C$, set $C^{\infty}=\{c \mid c \in C$ and $\nexists r \in R$ such that $(\mathcal{I}, \gamma) \vdash c \Rightarrow r\}$; if the following holds:

$c \in C^{\infty}$ implies that $c \Rightarrow \infty$ is the consequence of a rule where, for all premises of shape

$c^{\prime} \Rightarrow \infty, c^{\prime} \in C^{\infty}$ and, for all premises of shape $c^{\prime} \Rightarrow r$, with $r \in R,(\mathcal{I}, \gamma) \vdash c^{\prime} \Rightarrow r$

then $c \in C^{\infty}$ implies $(\mathcal{I}, \gamma) \vdash c \Rightarrow \infty$.

Proof. We consider the set $\mathcal{S}=\left\{c \Rightarrow \infty \mid c \in C^{\infty}\right\} \cup\{c \Rightarrow r \mid r \in R,(\mathcal{I}, \gamma) \vdash c \Rightarrow r\}$, and prove that $\mathcal{S} \subseteq \operatorname{Gen}(\mathcal{I}, \gamma)$ by bounded coinduction (Theorem 2.3).

- $\mathcal{S} \subseteq \operatorname{Ind}\left(\mathcal{I}_{\sqcup \gamma}\right)$. Indeed, for each $c \Rightarrow \infty \in \mathcal{S}, c \Rightarrow \infty$ is a direct consequence of the coaxiom, and for each $c \Rightarrow r \in \mathcal{S}$, with $r \in R$, since $(\mathcal{I}, \gamma) \vdash c \Rightarrow r$ holds, $c \Rightarrow r$ has a finite proof tree in $\mathcal{I}_{\sqcup \gamma}$ by definition.

- $\mathcal{S} \subseteq F_{\mathcal{I}}(\mathcal{S})$. Indeed:

- for each $c \Rightarrow \infty \in \mathcal{S}$, by hypothesis it is the consequence of a rule where, for all premises of shape $c^{\prime} \Rightarrow \infty, c^{\prime} \in C^{\infty}$ holds, hence $c^{\prime} \Rightarrow \infty \in \mathcal{S}$, and, for all premises of shape $c^{\prime} \Rightarrow r$, $(\mathcal{I}, \gamma) \vdash c^{\prime} \Rightarrow r$ holds, hence $c^{\prime} \Rightarrow r \in \mathcal{S}$ as well.

- for each $c \Rightarrow r \in \mathcal{S}$, with $r \in R$, by conservativity $\mathcal{I} \vdash c \Rightarrow r$ holds, hence it is the consequence of a rule where, for all premises, necessarily of shape $c^{\prime} \Rightarrow r^{\prime}$, with $r^{\prime} \in R$, $\mathcal{I} \vdash c^{\prime} \Rightarrow r^{\prime}$ holds, hence again by conservativity $c^{\prime} \Rightarrow r^{\prime} \in \mathcal{S}$.

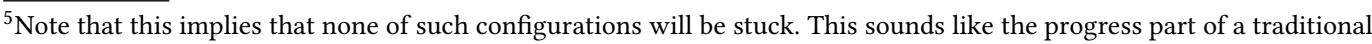
soundness result, and indeed one use of the divergence consistency principle in the following will be the proof of soundness of type systems.
} 
We will use this proof principle to obtain two results for the example of the $\lambda$-calculus: equivalence with the small-step semantics and soundness of the type system.

The definition of the small-step semantics is shown in Fig. 2.

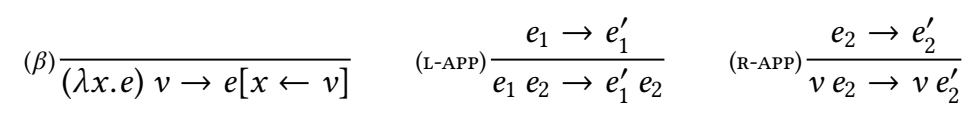

Fig. 2. $\lambda$-calculus: small-step semantics

We denote by $\rightarrow^{\star}$ the reflexive and transitive closure of the relation $\rightarrow$, and by InfSS the set of expressions which have an infinite sequence of small steps, which can be coinductively defined by: if $e^{\prime} \in \operatorname{InfSS}$ and $e \rightarrow e^{\prime}$, then $e \in$ InfSS.

The proof of equivalence needs the following standard lemma, see [Leroy and Grall 2009].

LEMMA 3.4. For all expressions e, exactly one of the following conditions holds: either e $\in$ InfSS or there exists $e^{\prime}$ such that $e \rightarrow^{\star} e^{\prime}$ and $e^{\prime} \nrightarrow$, that is, $\nexists e^{\prime \prime}$ such that $e^{\prime} \rightarrow e^{\prime \prime}$.

Theorem 3.5. $e \Rightarrow v$ iff $e \rightarrow^{\star} v$, and $e \Rightarrow \infty$ iff $e \in$ InfSS.

Proof. We prove separately the two statements.

(1) By Theorem 3.2, $e \Rightarrow v$ is derivable iff it is derivable in the inference system consisting of only rules (VAL) and (APP), hence the equivalence can be proved by standard inductive techniques. Shortly, $e \Rightarrow v$ implies $e \rightarrow^{\star} v$ can be proved by induction on such rules, whereas $e \rightarrow^{\star} v$ implies $e \Rightarrow v$ can be proved by arithmetic induction on the number of steps in $e \rightarrow^{\star} v$, plus the fact that $e \rightarrow e^{\prime}$ and $e^{\prime} \Rightarrow v$ implies $e \Rightarrow v$, which can be proved by induction on the rules which define $e \rightarrow e^{\prime}$.

(2)(a) $e \Rightarrow \infty$ implies $e \in$ InfSS can be proved by coinduction on the definition of InfSS.

(b) $e \in \operatorname{InfSS}$ implies $e \Rightarrow \infty$ can be proved by divergence consistency (Theorem 3.3). Set InfSS ${ }^{\infty}=\{e \mid e \in \operatorname{InfSS}$ and $\nexists v$ such that $e \Rightarrow v\}$ and note that InfSS ${ }^{\infty}=$ InfSS by point (1) and Lemma 3.4. Thanks to Theorem 3.3, it is enough to show that, for each $e \in \operatorname{InfSS^{\infty }}$, $e \Rightarrow \infty$ is the consequence of a rule where, for all premises of shape $e^{\prime} \Rightarrow \infty, e^{\prime} \in \operatorname{InfSS}^{\infty}$ and all premises of shape $e^{\prime} \Rightarrow v$ are derivable. The proof is by case analysis over $e$.

$v$ Empty case since values evaluate to themselves.

$x$ Empty case since variables cannot be reduced.

$e_{1} e_{2}$ If $e_{1} e_{2}$ has an infinite reduction sequence, then, by Lemma 3.4, there are three possible cases:

- $e_{1}$ has an infinite reduction sequence. In this case, $e_{1} e_{2} \Rightarrow \infty$ is the consequence of rule (L-Div) where, for the premise $e_{1} \Rightarrow \infty, e_{1} \in$ InfSS.

- $e_{1}$ has no infinite reduction sequence and $e_{2}$ has an infinite reduction sequence. In this case, it holds necessarily that $e_{1} \rightarrow^{\star} v$, otherwise $e_{1} e_{2}$ would be stuck, contradicting the hypothesis. Hence, $e_{1} e_{2} \Rightarrow \infty$ is the consequence of rule (R-Div) where, for the premise $e_{2} \Rightarrow \infty, e_{2} \in$ InfSS, and the premise $e_{1} \Rightarrow v$ is derivable by point (1).

- $e_{1}$ and $e_{2}$ have no infinite reduction sequence. In this case, it holds necessarily that $e_{1} \rightarrow^{\star} \lambda x . e$ and $e_{2} \rightarrow^{\star} v$, otherwise $e_{1} e_{2}$ would be stuck, contradicting the hypothesis, and $e[x \leftarrow v]$ has necessarily an infinite reduction sequence. Hence, $e_{1} e_{2} \Rightarrow \infty$ is the consequence of rule (APP) where, for the premise $e[x \leftarrow v] \Rightarrow \infty, e[x \leftarrow v] \in \operatorname{InfSS}$, and the premises $e_{1} \Rightarrow \lambda x$.e and $e_{2} \Rightarrow v$ are derivable by point (1). 
We illustrate now how to prove type soundness. We will consider simply-typed $\lambda$-calculus with recursive types, obtained by interpreting the production $\tau::=$ int $\mid \tau_{1} \rightarrow \tau_{2}$ coinductively. Enabling recursive types makes the calculus non-normalizing and allows one to write interesting programs such as $\Omega$ above.

The typing rules are recalled in Fig. 3. Type environments, written $\Gamma$, are finite maps from variables to types.

$$
\begin{gathered}
(\mathrm{T}-\mathrm{VAR}) \frac{(\mathrm{T}-\mathrm{INT})}{\Gamma \vdash x: \tau} \Gamma(x)=\tau \quad \frac{\Gamma \vdash \operatorname{int}}{\Gamma \vdash e_{1}: \tau^{\prime} \rightarrow \tau \quad \Gamma \vdash e_{2}: \tau^{\prime}} \\
\Gamma \vdash e_{1} e_{2}: \tau
\end{gathered}
$$

Fig. 3. $\lambda$-calculus: type system

The type system is sound when, informally, well-typed programs cannot "go wrong", following Robin Milner's slogan [Milner 1978]. In small-step style, as firstly formulated by Wright and Felleisen [Wright and Felleisen 1994], this can be expressed as follows: well-typed programs (closed terms) never reduce to terms which neither are values, nor can be further reduced (called stuck terms). The standard proof technique is to ensure soundness by subject reduction (well-typedness is preserved by reduction) and progress (a well-typed closed term is not stuck).

In big-step style with coaxioms, soundness of the type system can be directly expressed as follows: well-typed programs (closed terms) always reduce to a result (including divergence). Formally:

Theorem 3.6 (Soundness). If $\emptyset \vdash e: \tau$ for some $\tau$, then $e \Rightarrow v_{\infty}$ for some $v_{\infty}$.

Note that we cannot prove soundness by induction on the typing rules. Indeed, for rule (T-APP), by inductive hypothesis we would get that $e_{1}$ and $e_{2}$ reduce to a result. Even assuming canonical forms and substitution lemmas, in the case both are values, say, $\lambda x . e$ and $v$, respectively, we would be not able to apply rule (APP) since we have no inductive hypothesis on the term $e[x \leftarrow v]$.

Soundness can be shown, instead, as a direct consequence (rephrasing) of progress stated as below.

Theorem 3.7 (Progress). If $\emptyset \vdash e: \tau$ for some $\tau$, and $\nexists v$ such that $e \Rightarrow v$, then $e \Rightarrow \infty$.

Let $W T$ be the set of well-typed programs, that is, $W T=\{e \mid \emptyset \vdash e: \tau$ for some $\tau\}$, and $W T^{\infty}=\{e \mid e \in W T$ and $\nexists v$ such that $e \Rightarrow v\}$.

Thanks to Theorem 3.3, to prove progress it is enough to prove the following progress lemma.

Lemma 3.8 (Progress lemma). If $e \in W T^{\infty}$, then $e \Rightarrow \infty$ is the consequence of a rule where, for all premises of shape $e^{\prime} \Rightarrow \infty, e^{\prime} \in W T^{\infty}$, and all premises of shape $e^{\prime} \Rightarrow v$ are derivable.

The proof needs substitution and type preservation (the analogous of subject reduction) lemmas, which can be proved in the standard inductive way.

Lemma 3.9 (Substitution). If $x: \tau^{\prime} \vdash e: \tau$ and $\emptyset \vdash e^{\prime}: \tau^{\prime}$, then $\emptyset \vdash e\left[x \leftarrow e^{\prime}\right]: \tau$.

Proof. By induction on the typing rules.

LEMma 3.10 (Type PRESERVATION). If $\emptyset \vdash e: \tau$ and $e \Rightarrow v$, then $\emptyset \vdash v: \tau$.

Proof. By induction on the rules which define $e \Rightarrow v$.

Proof of Lemma 3.8 (Progress lemma). The proof is by case analysis over $e$. 
$v$ Empty case since values evaluate to themselves.

$x$ Empty case since variables have no type in the empty environment.

$e_{1} e_{2}$ The typing judgment $\emptyset \vdash e: \tau$ has been derived by rule (T-APP), hence $\emptyset \vdash e_{1}: \tau^{\prime} \rightarrow \tau$ and $\emptyset \vdash e_{2}: \tau^{\prime}$ hold as well. We have the following subcases:

- $\nexists v_{1}$ such that $e_{1} \Rightarrow v_{1}$. In this case, $e_{1} e_{2} \Rightarrow \infty$ is the consequence of rule (L-Div), where, in the premise $e_{1} \Rightarrow \infty, e_{1} \in W T^{\infty}$.

- $e_{1} \Rightarrow v_{1}$, and $\nexists v_{2}$ such that $e_{2} \Rightarrow v_{2}$. In this case, $e_{1} e_{2} \Rightarrow \infty$ is the consequence of rule (R-Div), where, in the premise $e_{2} \Rightarrow \infty, e_{2} \in W T^{\infty}$.

- $e_{1} \Rightarrow v_{1}$ and $e_{2} \Rightarrow v_{2}$. In this case, $e_{1} e_{2} \Rightarrow \infty$ is the consequence of rule (APP) with premise $e\left[x \leftarrow v_{2}\right] \Rightarrow \infty$. Moreover, $v_{1}=\lambda x . e$ and, by Lemma 3.10, $\emptyset \vdash \lambda x . e: \tau^{\prime} \rightarrow \tau$. This judgment has been derived by rule (T-ABs), hence $x: \tau^{\prime} \vdash e: \tau$, and, by Lemma 3.9, $\vdash \vdash e\left[x \leftarrow v_{2}\right]: \tau$. Moreover, $\nexists v$ such that $e\left[x \leftarrow v_{2}\right] \Rightarrow v$ since otherwise we could derive $e_{1} e_{2} \Rightarrow v$ as well, contradicting the hypothesis. Hence $e\left[x \leftarrow v_{2}\right] \in W T^{\infty}$.

\section{AN IMPERATIVE JAVA-LIKE LANGUAGE}

In this section we present a simple imperative Java-like language, to show in a more significant case the effectiveness of our approach. With the big-step style handling imperative features is easier than in small-step semantics; indeed, one does not have to introduce runtime terms for expressly modeling intermediate steps of computations in case of method calls and stack frames management, since these details are abstracted away by the big-step style.

The syntax is defined in Fig. 4. We write $\overline{c d}^{n}$ for the sequence $c d_{1} \ldots c d_{n}$, or simply $\overline{c d}$ when the length is not relevant, and analogously for other sequences. We assume sets of variables (parameters) $x$, including this, class names $c$, including object, field names $f$, and method names $m$.

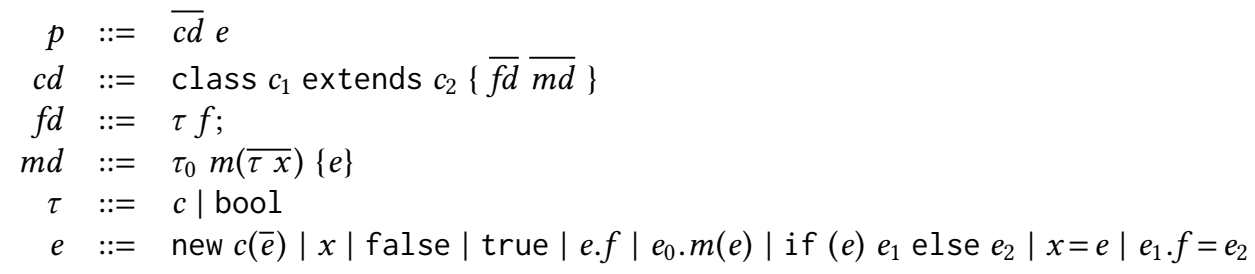

Fig. 4. FJ-like language: syntax

The language is a minimal imperative extension of Featherweight Java (FJ for short) [Igarashi et al. 2001], where variables and fields can be updated. Furthermore, conditional expressions and boolean constants have been added, while type casts have been omitted. For sake of simplicity, there are no statements, hence assignment is an expression with side effect, returning the value of its right-hand side expression.

We assume standard syntactic restrictions: inheritance is acyclic, names are distinct in class, method, field, and parameter declarations, object cannot be declared, class names other than object that appear in method headers and field declarations must be declared.

A program consists of a sequence of class declarations and a main expression. Types are either class names or the primitive type bool; however, for simplicity, bool is considered a predefined class which has no fields and methods, directly extends object, and has false and true as only instances.

A class declaration contains field and method declarations, and is equipped with an implicit constructor with parameters corresponding to all fields, in the same order as they are inherited 
and declared. Method declarations are standard. In the body, the target object can be accessed via the implicit parameter this, therefore all explicitly declared parameters must be different from this; moreover, this cannot be assigned. Expressions include instance creation, variables, boolean constants, field selection, method invocation, conditional expression, and variable and field update.

We define now the big-step semantics of the language; as for the case of $\lambda$-calculus we define a fully deterministic semantics based on the left-to-right call-by-value evaluation strategy. Values, results and evaluation contexts are defined in Fig. 5. We assume an infinite set of references $t$.

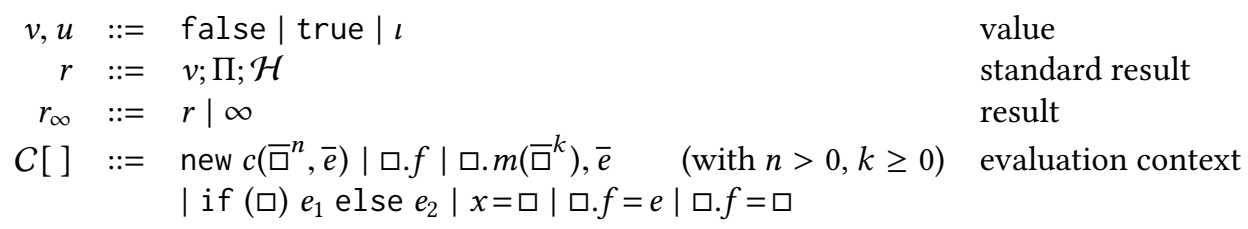

Fig. 5. FJ-like language: values, results and evaluation contexts

Values are either boolean constants or (object) references. Standard results are triples consisting of a value, an evaluation environment (stack frame) and a heap. The stack frame $\Pi$ of the method under execution is modeled as a finite partial map from variables (this and the explicit parameters of the method) to values. The heap $\mathcal{H}$ is a finite partial map from references to objects, which are are $\operatorname{obj}(c, \rho)$, where $c$ is the object's class and $\rho$ is a finite partial map from field names to values. We use the usual set-theoretic representation for finite maps, in particular for heaps $\mathcal{H}$ and maps $\rho$ from fields to values; the operator $\uplus$ denotes union of maps with disjoint domains.

Evaluation contexts contain one or more holes at depth ${ }^{6} 1$, and allow a more compact definition of divergence propagation (see rule (Div) in Fig. 6).

The big-step semantics is formalized by the judgment $\Pi ; \mathcal{H} ; e \Rightarrow r_{\infty}$, meaning that, in stack frame $\Pi$ and heap $\mathcal{H}$, expression $e$ either converges to value $v$, stack frame $\Pi^{\prime}$, and heap $\mathcal{H}^{\prime}$, or diverges, written $\Pi ; \mathcal{H} ; e \Rightarrow \infty$.

This big-step semantics is an instance of the general framework introduced in Sect. 3, where configurations are triples $\Pi ; \mathcal{H} ; e$, and standard results are triples $v ; \Pi ; \mathcal{H}$.

Heaps are required to be closed w.r.t. references: for all references $\iota$, if $\mathcal{H}(\iota)=o b j(c, \rho)$, then for all $f \in \operatorname{dom}(\rho), \rho(f) \in \operatorname{dom}(\mathcal{H}) \cup\{$ false, true $\}$. In other words, heaps cannot contain dangling references; this is a natural requirement for a language and a semantics where object references cannot be deallocated. In the claims in the rest of the paper we implicitly assume that heaps are closed w.r.t. references.

The semantic rules are defined in Fig. 6.

As usual, the judgment should be indexed over class declarations contained in the program, however for brevity we leave implicit such an index.

The straightforward definitions of the auxiliary functions fields, meth, and restore can be found in Fig. 7.

Rules are almost standard. The left-to-right call-by-value strategy affects the divergence propagation described in rules (DIv), (IFF), (IFT) and (INv). Indeed subexpressions are evaluated from left to right and the first subexpression that diverges makes the whole expression diverge, without considering remaining subexpressions.

Rule (Div) deals with all those cases where divergence changes the normal control flow of the program because not all subexpressions that should be evaluated are reached; all such situations

\footnotetext{
${ }^{6}$ Assuming that the root of a term has depth 0 .
} 


$$
\begin{aligned}
& (\mathrm{cOA}) \frac{\bullet}{\Pi ; \mathcal{H} ; e \Rightarrow \infty} \quad(\mathrm{DIv}) \frac{\forall i=1 . . n . \Pi_{i-1} ; \mathcal{H}_{i-1} ; e_{i} \Rightarrow v_{i} ; \Pi_{i} ; \mathcal{H}_{i} \quad \Pi_{n} ; \mathcal{H}_{n} ; e \Rightarrow \infty}{\Pi_{0} ; \mathcal{H}_{0} ; C\left[\bar{e}^{n}, e\right] \Rightarrow \infty} \\
& \text { (vAR) } \frac{}{\Pi ; \mathcal{H} ; x \Rightarrow v ; \Pi ; \mathcal{H}} \Pi(x)=v \\
& \text { (FAL) } \overline{\Pi ; \mathcal{H} ; \text { false } \Rightarrow \text { false; } \Pi ; \mathcal{H}} \quad \text { (тRU) } \overline{\Pi ; \mathcal{H} ; \text { true } \Rightarrow \text { true } \Pi ; \mathcal{H}} \\
& \text { (NEw) } \frac{\forall i=1 . . n . \Pi_{i-1} ; \mathcal{H}_{i-1} ; e_{i} \Rightarrow v_{i} ; \Pi_{i} ; \mathcal{H}_{i}}{\Pi_{0} ; \mathcal{H}_{0} ; \text { new } c\left(\bar{e}^{n}\right) \Rightarrow \iota ; \Pi_{n} ; \mathcal{H}_{n} \uplus\left\{\iota \mapsto o b j\left(c, \bar{f}^{n} \mapsto \bar{v}^{n}\right)\right\}} \text { fields }(c)=\overline{\tau f}^{n} \\
& \text { (IFT) } \frac{\Pi_{0} ; \mathcal{H}_{0} ; e \Rightarrow \text { true } ; \Pi_{1} ; \mathcal{H}_{1} \quad \Pi_{1} ; \mathcal{H}_{1} ; e_{1} \Rightarrow r_{\infty}}{\Pi_{0} ; \mathcal{H}_{0} ; \text { if }(e) e_{1} \text { else } e_{2} \Rightarrow r_{\infty}} \\
& \text { (IFF) } \frac{\Pi_{0} ; \mathcal{H}_{0} ; e \Rightarrow \text { false; } \Pi_{1} ; \mathcal{H}_{1} \quad \Pi_{1} ; \mathcal{H}_{1} ; e_{2} \Rightarrow r_{\infty}}{\Pi_{0} ; \mathcal{H}_{0} ; \text { if }(e) e_{1} \text { else } e_{2} \Rightarrow r_{\infty}} \\
& \text { (FLD) } \frac{\Pi_{0} ; \mathcal{H}_{0} ; e \Rightarrow \iota ; \Pi_{1} ; \mathcal{H}_{1} \quad \mathcal{H}_{1}(\iota)=o b j(c, \rho \uplus\{f \mapsto v\})}{\Pi_{0} ; \mathcal{H}_{0} ; e . f \Rightarrow v ; \Pi_{1} ; \mathcal{H}_{1}}
\end{aligned}
$$

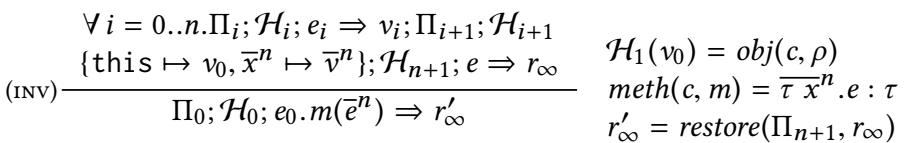

$$
\begin{aligned}
& \text { (vAs) } \frac{\Pi_{0} ; \mathcal{H}_{0} ; e \Rightarrow v ; \Pi_{1} \uplus\{x \mapsto u\} ; \mathcal{H}_{1}}{\Pi_{0} ; \mathcal{H}_{0} ; x=e \Rightarrow v ; \Pi_{1} \uplus\{x \mapsto v\} ; \mathcal{H}_{1}} \\
& \Pi_{0} ; \mathcal{H}_{0} ; e_{1} \Rightarrow \imath ; \Pi_{1} ; \mathcal{H}_{1} \\
& \text { (FAs) } \frac{\Pi_{1} ; \mathcal{H}_{1} ; e_{2} \Rightarrow v ; \Pi_{2} ; \mathcal{H}_{2}}{\Pi_{0} ; \mathcal{H}_{0} ; e_{1} \cdot f=e_{2} \Rightarrow v ; \Pi_{2} ; \mathcal{H} \uplus\{\iota \mapsto o b j(c, \rho \uplus\{f \mapsto v\})\}} \mathcal{H}_{2}=\mathcal{H} \uplus\{\iota \mapsto o b j(c, \rho \uplus\{f \mapsto u\})\}
\end{aligned}
$$

Fig. 6. FJ-like language: big-step semantics

are captured by the evaluation contexts defined in Fig. 5. Recall that the multiple arguments to an evaluation context are given in the order they should be evaluated in left-to-right call-by-value evaluation strategy.

In rule (inv), auxiliary function restore is used for restoring the stack frame of the caller, that is, the stack frame $\Pi_{n+1}$ obtained after evaluating the last argument $e_{n}$ of the method invocation, in case the evaluation of the body of the invoked method terminates; if the method diverges, then divergence is propagated, hence restore $\left(\Pi_{n+1}, \infty\right)=\infty$.

As already noted in Sect. 3, the unique coaxiom ( $\mathrm{COA}$ ) is crucial to ensure that divergence is captured in a proper way, indeed it allows infinite proofs only for divergence.

For instance, assuming the following class declaration

class $C$ extends Object $\{$ bool $m()\{$ this.m() $\}$ \}

and the expression $e=$ new $\mathrm{C}() \cdot \mathrm{m}()$, there exist no value $v$, stack frame $\Pi$, and heap $\mathcal{H}$ s.t. $\emptyset ; \emptyset ; e \Rightarrow$ $v ; \Pi ; \mathcal{H}$, even though the class $\mathrm{C}$ and the expression $e$ are well-typed. 


$$
\begin{aligned}
& \text { fields }\left(c^{\prime}\right)=\overline{\tau f}^{m} \\
& \text { class } c \text { extends } c^{\prime}\left\{{\overline{\tau^{\prime} f^{\prime}}}^{n} \overline{m d}\right\} \\
& \bar{f}^{m} \cap{\overline{f^{\prime}}}^{n}=\emptyset \\
& \overline{\text { fields(Object })=\epsilon \quad \text { fields }(c)=\overline{\tau^{\prime} f^{\prime}}, \overline{\tau f}} \\
& \frac{\text { class } c \text { extends } c^{\prime}\left\{\overline{f d} \overline{m d} \tau m(\overline{\tau x})\{e\} \overline{m d^{\prime}}\right\}}{m e t h(c, m)=\overline{\tau x} \cdot e: \tau} \\
& \operatorname{meth}\left(c^{\prime}, m\right)=\overline{\tau x} \cdot e: \tau \\
& \text { class } c \text { extends } c^{\prime}\{\overline{f d} \overline{m d}\} \\
& m \text { not declared in } \overline{m d} \\
& \operatorname{meth}(c, m)=\overline{\tau x} \cdot e: \tau \\
& \operatorname{restore}\left(\Pi, r_{\infty}\right)= \begin{cases}\infty & \text { if } r_{\infty}=\infty \\
v ; \Pi ; \mathcal{H} & \text { if } r_{\infty}=v ; \Pi^{\prime} ; \mathcal{H}\end{cases}
\end{aligned}
$$

Fig. 7. Definition of auxiliary functions fields, meth, and restore

On the other hand, if we consider the coinductive interpretation of the semantics rules (and disregard the coaxiom), we have that $\emptyset$; $\emptyset$; new $\mathrm{C}() \cdot \mathrm{m}() \Rightarrow r_{\infty}$ is derivable for all possible results $r_{\infty}$, since we can always build an infinite proof tree for the judgment, as depicted in Fig. 8.

$$
(\mathrm{INV}) \frac{(\mathrm{NEW}) \frac{(\mathrm{VAR})}{\emptyset ; \emptyset ; \text { new } \mathrm{C}() \Rightarrow \imath ; \emptyset ; \mathcal{H}} \quad(\mathrm{INV}) \frac{\vdots}{\Pi \text { this } \Rightarrow l ; \Pi ; \mathcal{H}} \quad(\mathrm{INV}) \frac{1}{\Pi ; \mathcal{H} ; \text { this.m( }) \Rightarrow r_{\infty}}}{\Pi ; \mathcal{H} ; \text { this.m( }) \Rightarrow r_{\infty}}
$$

where $\Pi=\{$ this $\mapsto \iota\}, \mathcal{H}=\{\iota \mapsto \operatorname{obj}(\mathrm{C}, \emptyset)\}$

Fig. 8. Tree for $\emptyset ; \emptyset$; new $C() \cdot m() \Rightarrow r_{\infty}$

Indeed, this example is analogous to the $\Omega$ example for the $\lambda$-calculus, and, analogously, using ( $\mathrm{COA}$ ) infinite trees are only allowed if all nodes in the tree are provable with a finite tree using ( $\mathrm{COA})$ as axiom, therefore the tree in Fig. 8 is valid only when $r_{\infty}=\infty$.

The following example shows that an ill-typed expression with a divergent subexpression correctly diverges, analogously to the example $\Omega(00)$ for the $\lambda$-calculus.

Let us consider again class C: The expression $e=\mathbf{i f}($ new $\mathrm{C}() \cdot \mathrm{m}())$ true.m() else true.m() diverges according to the proof tree in Fig. 9, because the guard is evaluated first, and since it diverges, other subexpressions are not considered.

We show now an example which illustrates the difference with the approach in [Ancona 2011, 2012, 2014], where, in order to have soundness of the type system, non well-founded values (that is, values corresponding to infinite trees) were considered. In an imperative language, this would correspond to allowing heaps to have infinite domain, since the abstract structure of values can be recovered from the heap. The approach with coaxioms allows us to be more restrictive, requiring heaps to be finite partial maps, since divergence is captured by the special result $\infty$.

Let us consider for instance the following class declarations: 


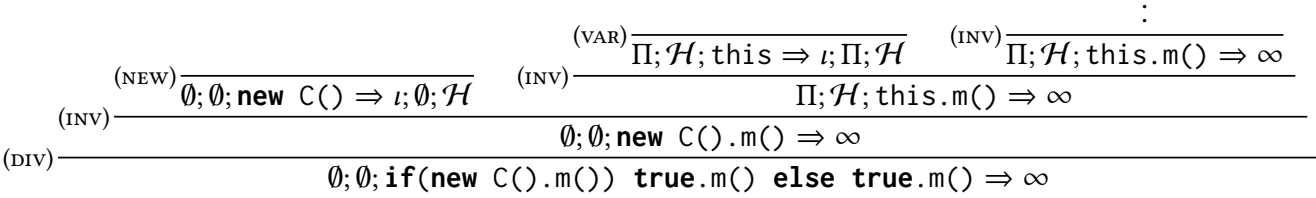

where $\Pi=\{$ this $\mapsto \iota\}, \mathcal{H}=\{\iota \mapsto \operatorname{obj}(\mathrm{C}, \emptyset)\}$

Fig. 9. Tree for $\emptyset ; \emptyset$; if (new $C() \cdot m())$ true.m() else true.m() $\Rightarrow \infty$

class $M$ extends $\operatorname{Object}\{L m()\{$ new $L($ this.m( $))\}\}$

class $L$ extends $\operatorname{object}\{L n ;\}$

$$
\nabla=(\mathrm{INV}) \frac{(\mathrm{NEW}) \overline{\emptyset ; \emptyset ; \text { new } \mathrm{M}() \Rightarrow \imath ; \emptyset ; \mathcal{H}} \quad \nabla^{\prime}}{\emptyset ; \emptyset ; \text { new } \mathrm{M}() \cdot \mathrm{m}() \Rightarrow \infty}
$$

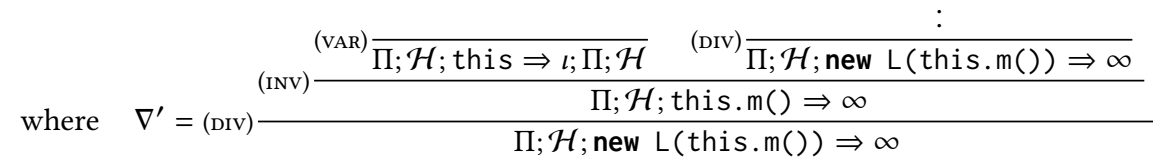

$$
\begin{aligned}
& \Pi=\{\text { this } \mapsto \iota\}, \mathcal{H}=\{\iota \mapsto \operatorname{obj}(\mathrm{C}, \emptyset)\}
\end{aligned}
$$

Fig. 10. Proof tree $\nabla$ for $\emptyset ; \emptyset$; new $M() \cdot m() \Rightarrow \infty$

The infinite but regular proof tree for the judgment $\emptyset ; \emptyset$; new $M() \cdot m() \Rightarrow \infty$ is shown in Fig. 10 . This expression tries to build the regular object $X=$ new $L(X)$, and this causes the expression to diverge.

To conclude this section we state in a more formal way the relationship between the standard big-step semantics, that is, the inductive interpretation of the inference rules, and the interpretation generated by coaxioms. That is, we show that the big-step semantics in Fig. 6 is conservative according to Def. 3.1.

Theorem 4.1 (Conservativity). If $\Pi ; \mathcal{H} ; e \Rightarrow r_{\infty}$ holds then $r_{\infty} \neq \infty$ if and only if the judgment has a finite proof tree.

Proof. Let us denote by $(\mathcal{I}, \gamma)$ the generalized inference system defining $\Pi ; \mathcal{H} ; e \Rightarrow r_{\infty}$ in Fig. 6 , and by $\mathcal{I}_{\sqcup \gamma}$ the (standard) inference system obtained considering ( $\left.\mathrm{COA}\right)$ as axiom. If $\Pi ; \mathcal{H} ; e \Rightarrow r_{\infty}$ is derivable in $(\mathcal{I}, \gamma)$, by definition each judgment of the proof tree, including the judgment itself, must also have a finite proof tree in $\mathcal{I}_{\sqcup \gamma}$. Noting that a rule's consequence is a divergent judgment iff at least one of the premises is divergent (rules (DIv), (IFT), (IFF) and (INv)), by induction on the depth of the tree it can be shown that $r_{\infty}=\infty$ iff we use (COA) in the tree. It follows that $r_{\infty} \neq \infty$ iff we do not use (СоA) in the finite proof tree in $\mathcal{I}_{\sqcup \gamma}$. Hence, this proof tree is a finite proof tree in $(\mathcal{I}, \gamma)$ as well, as needed.

In other words, this theorem states that the set of convergent judgments corresponds exactly to the set inductively defined by the rules of the big-step semantics. This means that we can reason by induction when we have to define functions or to prove properties on convergent judgments. 


$$
\begin{aligned}
& \text { (T-PRO) } \frac{\forall i=1 . . n . \vdash c d_{i}: \diamond \emptyset \vdash e: \tau}{\vdash \overline{c d}^{n} e: \diamond} \quad \text { (T-CLA) } \frac{\forall i=1 . . k . c \vdash m d_{i}: \diamond \quad \text { fields }(c) \text { defined }}{\vdash \text { class } c \text { extends } c^{\prime}\left\{\overline{f d}^{k} \overline{m d}^{k}\right\}: \diamond} \\
& \text { (Т-MET) } \frac{\text { this : } c, \overline{x: \tau^{n}} \vdash e: \tau \quad \tau \leq \tau_{0} \quad \operatorname{override}(c, m)}{c \vdash \tau_{0} m\left(\overline{\tau x}^{n}\right)\{e\}: \diamond} \\
& \text { (T-VAR) } \frac{\Gamma(x)=\tau}{\Gamma \vdash x: \tau} \quad \text { (T-FAL) } \overline{\Gamma \vdash \text { false : bool }} \quad \text { (T-TRU) } \overline{\Gamma \vdash \text { true : bool }} \\
& (\mathrm{T-NEW}) \frac{\forall i=1 . . n . \Gamma \vdash e_{i}: \tau_{i} \quad \text { fields }(c)={\overline{\tau^{\prime} f}}^{n} \quad \forall i=1 . . n . \tau_{i} \leq \tau_{i}^{\prime}}{\Gamma \vdash \operatorname{new} c\left(\bar{e}^{n}\right): c} \\
& \text { (T-IF) } \left.\frac{\Gamma \vdash e: \text { bool } \quad \Gamma \vdash e_{1}: \tau_{1} \quad \Gamma \vdash e_{2}: \tau_{2}}{\Gamma \vdash \text { if }(e) e_{1} \text { else } e_{2}: \vee\left(\tau_{1}, \tau_{2}\right)} \quad \text { (T-FLD }\right) \frac{\Gamma \vdash e: c \quad \text { fields }(c)=\bar{\tau} \bar{f} \quad 1 \leq i \leq n}{\Gamma \vdash e \cdot f_{i}: \tau_{i}} \\
& (\mathrm{~T}-\mathrm{INV}) \frac{\forall i=0 . . n . \Gamma \vdash e_{i}: \tau_{i} \quad \operatorname{meth}\left(\tau_{0}, m\right)={\overline{\tau^{\prime} x}}^{n} . e: \tau \quad \forall i=1 . . n . \tau_{i} \leq \tau_{i}^{\prime}}{\Gamma \vdash e_{0} \cdot m\left(\bar{e}^{n}\right): \tau} \\
& (\mathrm{T}-\mathrm{VAS}) \frac{\Gamma \vdash e: \tau \quad \Gamma(x)=\tau^{\prime} \quad \tau \leq \tau^{\prime}}{\Gamma \vdash x=e: \tau} \\
& \text { (T-FAs) } \frac{\Gamma \vdash e_{1}: c \quad \text { fields }(c)=\overline{\tau f}^{n} \quad \Gamma \vdash e_{2}: \tau \quad \tau \leq \tau_{i} \quad 1 \leq i \leq n}{\Gamma \vdash e_{1} \cdot f_{i}=e_{2}: \tau}
\end{aligned}
$$

Fig. 11. Nominal type system

Moreover this theorem implies that this big-step semantics is an instantiation of the general framework introduced in Sect. 3, therefore we can apply the divergence consistency principle.

\section{SOUNDNESS OF THE TYPE SYSTEM}

This section briefly defines the standard type system of the language based on nominal types and then shows its soundness applying the technique described in Sect. 3. All definitions are pretty standard, and have been included for sake of completeness.

The typing rules can be found in Fig. 11. A type environment $\Gamma$ is a finite sequence $\overline{x: \tau}$, where all variables $\bar{x}$ are distinct, denoting a finite partial function mapping variables to types $(\emptyset$ denotes the empty type environment, $\operatorname{dom}(\Gamma)$ the domain of $\Gamma$ ). Rules (T-PRo), (T-СLA), and (T-MET) define well-typed programs, classes, and methods, respectively. The other rules define well-typed expressions w.r.t. a given type environment. Let us recall that, as for the operational semantics, all typing judgments are implicitly indexed over a class table.

Besides functions fields and meth, introduced in the big-step semantics, the typing rules are based on the following auxiliary functions/operators, whose straightforward definition can be found in Fig. 12.

For the standard subtyping relation $\leq$ between nominal types, we assume that bool $\leq$ object, that is, that Boolean values are convertible to instances of class Object. When $c$ contains the declaration $\tau m\left(\overline{\tau x}^{n}\right)\{e\}$, override $(c, m)$ holds iff for the direct superclass $c^{\prime}$ of $c$, either meth $\left(c^{\prime}, m\right)$ is undefined, or $m e t h\left(c^{\prime}, m\right)={\overline{\tau^{\prime} x^{\prime}}}^{n}$.e $: \tau^{\prime},{\overline{\tau^{\prime}}}^{n} \leq \bar{\tau}^{n}$, and $\tau \leq \tau^{\prime}$. The join operator $\vee$ computes the least upper bound $\vee\left(\tau_{1}, \tau_{2}\right)$ of two types $\tau_{1}$ and $\tau_{2}$ (this is always defined since inheritance is single, and bool is a subtype of the top type object). 


$$
\begin{aligned}
& c^{\prime} \leq c^{\prime \prime} \\
& \text { (воX) } \overline{\text { bool } \leq \text { object }} \quad \text { (REF) } \frac{}{\tau \leq \tau} \quad \text { (INH) } \frac{\text { class } c \text { extends } c^{\prime}\{\ldots\}}{c \leq c^{\prime \prime}} \\
& \text { class } c \text { extends } c^{\prime}\{\ldots\} \\
& \operatorname{meth}\left(c^{\prime}, m\right)={\overline{\tau^{\prime} x^{\prime}}}^{n} \cdot e^{\prime}: \tau^{\prime} \\
& \text { class } c \text { extends } c^{\prime}\{\ldots\} \\
& \text { meth }\left(c^{\prime}, m\right) \text { undefined } \\
& \text { override }(c, m) \\
& \frac{\tau_{2} \leq \tau_{1}}{\vee\left(\tau_{1}, \tau_{2}\right)=\tau_{1}} \quad \frac{\tau_{1} \leq \tau_{2}}{\vee\left(\tau_{1}, \tau_{2}\right)=\tau_{2}} \\
& \begin{array}{l}
\vee\left(c_{1}^{\prime}, c_{2}^{\prime}\right)=c \\
c_{1} \text { extends } c_{1}^{\prime}
\end{array} \\
& \tau_{1}=\text { bool, } \tau_{2} \neq \text { bool or } \\
& \frac{\tau_{1} \neq \text { bool, } \tau_{2}=\text { bool }}{\vee\left(\tau_{1}, \tau_{2}\right)=\text { object }} \\
& \operatorname{meth}(c, m)=\overline{\tau x}^{n} . e: \tau \\
& \frac{{\overline{\tau^{\prime}}}^{n} \leq \bar{\tau}^{n} \text { and } \tau \leq \tau^{\prime}}{\text { override }(c, m)} \\
& \text { class } c_{1} \text { extends } c_{1}^{\prime}\{\ldots\} \\
& \text { class } c_{2} \text { extends } c_{2}^{\prime}\{\ldots\} \\
& \frac{c_{1} \not \leq c_{2}, c_{2} \not \leq c_{1}}{\vee\left(c_{1}, c_{2}\right)=c}
\end{aligned}
$$

Fig. 12. Definition of subtyping, override, and $\vee$

$$
\begin{gathered}
(\mathrm{v}-\mathrm{cOAx}) \frac{\bullet}{\mathcal{H} \vdash v: \tau} \quad(\mathrm{v}-\mathrm{Bool}-1) \frac{v=\text { false or } v=\text { true }}{\mathcal{H} \vdash v: \text { Object }} \quad(\mathrm{v}-\mathrm{BooL}-2) \frac{v=\text { false or } v=\text { true }}{\mathcal{H} \vdash v: \text { bool }} \\
\left(\mathrm{v} \text {-овJ) } \frac{\mathcal{H}(\iota)=\operatorname{obj}\left(c,\left\{\bar{f}^{n} \mapsto \bar{v}^{n}\right\}\right) \quad \forall i=1 . . n . \mathcal{H} \vdash v_{i}: \tau_{i} \quad c \leq c^{\prime} \quad \text { fields }(c)=\overline{\tau f}^{n}}{\mathcal{H} \vdash \iota: c^{\prime}}\right.
\end{gathered}
$$

Fig. 13. Type assignment for values

Let us now formalize and prove the main claim of soundness. We first introduce the judgment $\mathcal{H} \vdash v: \tau$ assigning types to values w.r.t. a heap; such a relation corresponds to the intended semantics of types as set of values [Ancona and Corradi 2014], and is defined by the rules in Fig. 13. Since a heap can contain a loop, rules must be interpreted coinductively; this is specified by the coaxiom (v-COAx) for which any typing is allowed, hence no judgment is filtered out, and, hence, the unbounded greatest fixed point of the definition is considered. The judgment supports subsumption to guarantee the validity of type preservation (Lemma A.10); subsumption is ensured by the side condition $c \leq c^{\prime}$ in rule (v-овJ), and by rule (v-BooL-1) needed to derive that a boolean value has type object, besides type bool (rule (v-воo--2)).

Agreement of evaluation environments and heaps with type environments is defined on top of the judgment $\mathcal{H} \vdash v: \tau$.

Definition 5.1. The evaluation environment $\Pi$ and heap $\mathcal{H}$ agree with the type environment $\Gamma$ (written $(\Pi ; \mathcal{H}): \Gamma)$ iff $\operatorname{dom}(\Gamma) \subseteq \operatorname{dom}(\Pi)$, and, for all $x \in \operatorname{dom}(\Gamma), \mathcal{H} \vdash \Pi(x): \Gamma(x)$ holds.

The main claim of soundness is the following.

Theorem 5.2 (Soundness). Let $\overline{c d}$ be well-typed class declarations. If $\Gamma \vdash e: \tau$ in $\overline{c d}$, and $(\Pi ; \mathcal{H}): \Gamma$, then there exists $r_{\infty}$ s.t. $\Pi ; \mathcal{H} ; e \Rightarrow r_{\infty}$.

Clearly, as a particular case of the theorem above, we get that if $\overline{c d} e$ is a well-typed program, according to rule (т-Pro), then there exists $r_{\infty}$ s.t. $\emptyset ; \emptyset ; e \Rightarrow r_{\infty}$.

To prove Theorem 5.2 we first rephrase it in the following progress theorem. 
Theorem 5.3 (Progress). Let $\overline{c d}$ be well-typed class declarations. If $\Gamma \vdash e: \tau$ in $\overline{c d},(\Pi ; \mathcal{H}): \Gamma$ and $\nexists r$ such that $\Pi ; \mathcal{H} ; e \Rightarrow r$, then $\Pi ; \mathcal{H} ; e \Rightarrow \infty$ holds.

We prove the theorem above by the technique described in Sect. 3. Indeed, it follows from Theorem 3.3 and the following progress lemma. Set $W T=\{\Pi ; \mathcal{H} ; e \mid \exists \Gamma, \tau . \Gamma \vdash e: \tau$ and $(\Pi ; \mathcal{H}): \Gamma\}$ and $W T^{\infty}=\{\Pi ; \mathcal{H} ; e \mid \Pi ; \mathcal{H} ; e \in W T$ and $\nexists r . \Pi ; \mathcal{H} ; e \Rightarrow r\}$.

Lemma 5.4 (Progress lemma). If $\Pi ; \mathcal{H} ; e \in W T^{\infty}$ then $\Pi ; \mathcal{H} ; e \Rightarrow \infty$ is the consequence of a rule where, for all premises of shape $\Pi^{\prime} ; \mathcal{H}^{\prime} ; e^{\prime} \Rightarrow \infty, \Pi^{\prime} ; \mathcal{H}^{\prime} ; e^{\prime} \in W T^{\infty}$, and all premises of shape $\Pi^{\prime} ; \mathcal{H}^{\prime} ; e^{\prime} \Rightarrow r$ are derivable.

The proof of the progress lemma is provided in the Appendix.

\section{RELATED WORK}

Related work includes papers proposing techniques for proving type soundness of type systems, as well as investigation of more convenient ways to define the operational semantics of languages.

Milner and Tofte [Milner and Tofte 1991] have been among the first who employed coinduction for proving properties of a type system for a simple functional language; coinduction is used to reason on closures corresponding to recursive functions. However, they only prove consistency of static and dynamic semantics, which is weaker than type soundness, and, indeed, their semantic rules are interpreted inductively, and their semantics does not capture diverging evaluations.

In the work by Ernst et al. [Ernst et al. 2006] type soundness of a calculus of virtual classes is proved in terms of a big-step operational semantics thanks to a coverage lemma ensuring that errors do not prevent expressions from evaluating to a result. Such a result is achieved by introducing a finite evaluation relation indexed over natural numbers which allows managing divergence in a similar way as in the approaches based on approximating big-step semantics [Ancona 2014], functional big-step semantics [Owens et al. 2016] and definitional interpreters [Amin and Rompf 2017] with step counters (see details below regarding these approaches). A terminating expression is one for which there exists a natural number $n$ such that the finite evaluation indexed by $n$ returns a value (which may include also the usual runtime errors); the solution proposed by Ernst et al. requires introducing extra rules for dealing with runtime errors generation and propagation, hence it shares redundancy issues with other approaches.

Kusmierek and Bono [Kusmierek and Bono 2010] propose a different approach and prove type soundness in terms of an inductive big-step operational semantics; their proposal is centered on the idea of tracing the intermediate steps of a program execution with a partial derivation-search algorithm which deterministically computes the value and the proof tree of evaluation judgments. Similar approaches, although their corresponding semantics are not deterministic, are those of Ager [Ager 2004] and Stoughton [Stoughton 1997].

Ancona studies the big-step operational semantics of a simple non imperative Java-like language [Ancona 2012] by interpreting the standard rules coinductively; he shows that if the set of values includes also non well-founded ones, then it is possible to correctly express and prove a type soundness claim. Anyway, the proposed proof technique is rather complex, and the semantics fails to properly capture divergence; for instance, there exist divergent programs for which the coevaluation judgment returns any value, thus exhibiting a fully non deterministic behavior. In a followup paper [Ancona 2014], he proposes a simplified approach based on the notion of approximating big-step semantics, based on an inductively defined evaluation judgment able to approximate coevaluation; in this way, the proof of type soundness can be simplified.

Cousot and Cousot [Cousot and Cousot 1992] were the first to propose a coinductive big-step definition of divergence for the call-by-value $\lambda$-calculus, by introducing two different judgments 
defined in a stratified way: terminating computations are modeled by a standard inductive judgment, while divergence is characterized by a coinductive judgment, whose definition depends from the inductive judgments.

The approach has the drawback that some metarules have to be duplicated for both judgments because the two inference systems require two different interpretations, one inductive and the other coinductive; in the simple case of $\lambda$-calculus, only the metarule for function application is duplicated, but for more complex languages several rules need to be repeated; for instance, for the imperative Java-like language formalized in Sect. 4, rules (IFF), (IFT) and (INv) should be duplicated.

This redundancy is avoided in followup work [Cousot and Cousot 2009] based on the notion of bi-inductive semantics, where a unique inference system is provided for modeling both terminating and diverging computations; the semantics of the inference system is neither the standard inductive nor the coinductive ones, but is defined in terms of the least fixed point with respect to a nonstandard ordering. It is not so clear how this approach can easily scale to more complex languages, such as the imperative object-oriented language we consider here.

In their work Leroy and Grall [Leroy and Grall 2009] analyze two kinds of big-step operational semantics for the call-by-value $\lambda$-calculus, study their relationships with the small-step and denotational semantics, and their suitability for type soundness and compiler correctness proofs.

One semantics is based on the approach followed by Cousot and Cousot [Cousot and Cousot 1992] with two stratified judgments, one inductive for terminating computations, and the other coinductive, to capture divergence, defined on top of the inductive one; as already observed previously, such an approach suffers from redundancy, since some metarules have to be duplicated for both judgments.

The other semantics is obtained by simply interpreting coinductively the standard evaluation rules for terminating evaluations. However, this semantics fails to capture divergence, as happens for the approach followed by Ancona [Ancona 2012, 2014]; furthermore, differently from the work by Ancona, type soundness cannot be proved because the judgment is defined only for well-founded values, and thus, there exist well-typed divergent terms for which the judgment returns no well-founded value.

Nakata and Uustalu [Nakata and Uustalu 2009, 2010a] define a coinductive trace-based bigstep semantics for an imperative language. Traces keep track of all intermediate states of the computation; since traces are allowed to be infinite, and the judgment is defined coinductively, the semantics captures divergence; in a followup paper [Nakata and Uustalu 2010b], they define several big-step semantics for a simple imperative language with interactive $\mathrm{I} / \mathrm{O}$ which are based on resumptions (trees representing possible runs of a program) and require nesting inductive definitions in coinductive ones.

Pretty-big-step semantics has been proposed by Charguéraud [Charguéraud 2013] with the main aim of solving the problem of duplicated rules which prevents several approaches from scaling up to realistic programming languages; this is achieved by decomposing complex metarules into simpler ones with less premises, and by introducing intermediate runtime terms to avoid undesired overlapping between the metarules; anyway, as in previous approaches [Cousot and Cousot 1992; Leroy and Grall 2009], to model divergence, a separate coinductively defined judgment needs to be introduced, with similar problems of duplication. To assess scalability a formalization is provided for a non trivial subset of Caml, including exceptions and imperative features.

There exist several proposals based on Reynolds' original idea of definitional interpreters. Danielsson [Danielsson 2012] shows how big-step or small-step operational semantics for lambda-calculi can be defined as total, computable functions with the coinductive partiality monad.

Owens et al. [Owens et al. 2016] propose functional big-step semantics as a way to give a more readable account of a semantics; in their approach big-step semantics consists of a total function 
rather than of a judgment defined by a set of inference rules. To ensure totality even in the presence of divergent computations, a clock is introduced to be decreased in certain recursive calls and in loops; in this way, a non terminating computation always returns a timeout for any initial value of the clock, hence induction can be used to reason on divergence, as it is the usual case for small-step semantics, and as happens also for the approximating big-step semantics [Ancona 2014], and definitional interpreters with a step counter; furthermore, I/O and non-determinism can be supported with the use of an oracle. Besides readability, authors claim that functional big-step semantics can ease mechanized proofs based on rewriting; to this aim, they include a proof showing that a compiler implements one of the allowed input/output behaviors specified by a non-deterministic semantics for the source language.

Amin and Rompf [Amin and Rompf 2017] exploit the possibility of defining operational semantics with high-level definitional interpreters as total functions parametric in a step counter, and propose purely inductive proof strategies for type soundness properties that can scale to advanced polymorphic type systems as System $\mathrm{F}_{<:}$.

Reachability logic has been employed [Stefanescu et al. 2016] to prove divergence without touching the semantics of the language at all (although working with a small-step semantics). The formal relationship with our approach deserves further investigation; overall, the reachability logic approach is focused on the more ambitious aim of expressing in the same formalism both language semantics and specifications of properties to be proved, while our main concern is investigating new techniques to define language semantics in better ways.

For what concerns core calculi to model the static and dynamic semantics of Java, or to investigate more advanced type systems, there exist several proposals, although most of them consider non imperative subsets of Java which are variations of FJ [Igarashi et al. 2001]. Anyway, all of them prove type soundness in terms of a small-step operational semantics.

Among imperative calculi for Java, it is worth mentioning the work of Drossopoulou and Eisenbach [Drossopoulou et al. 1999], which covers a large part of Java, including exceptions, but not multithreading, and Welterweight Java [Östlund and Wrigstad 2010] which models Java's thread-based concurrency and lock-based synchronization. Also for the imperative case, all calculi are based on a small-step operational semantics.

\section{CONCLUDING DISCUSSION AND FUTURE WORK}

We have shown that coaxioms can be conveniently adopted in the context of operational semantics to express terminating and divergent computations in a compact way by a unique judgment. We have demonstrated the idea on big-step semantics, leaving for future work to experiment with other semantic approaches, such as small-step, CHAM, reduction semantics with evaluation contexts, continuation-based, etc.

The approach is based on a coevaluation judgment defined by a generalized inference system with coaxioms. The metarules for defining the coevaluation judgment can be obtained by adapting and extending in the following way the metarules that would define the standard inductive big-step evaluation judgment (for convenience we call them standard metarules):

- the set of results has to be extended with a single value representing divergence (denoted by $\infty$ in this paper);

- accordingly, in all standard metarules, meta-variables referring to results must range over the extended set of results to correctly take into account divergence. In simple cases, as shown for the $\lambda$-calculus, no further adaptation of the standard metarules is required, while for more complex languages, as the imperative FJ-like language defined in Sect. 4, some metarule (in the concrete example, only metarule (inv) for method invocation) may need 
some adjustment; this happens whenever the result that has to be returned in case of a terminating computation does not coincide with any of the results returned in the premises of the metarule. For instance, this happens in Sect. 4 because results contain stack frames, and in metarule (INv) the stack frame of the caller has to be restored.

- a new metarule for divergence propagation has to be added, to manage all those cases where divergence modifies the normal control flow (that is, the control flow specified by the standard rules) because not all subexpressions that should be evaluated are reached; to avoid replication, such a metarule can be defined in terms of a set of evaluation contexts that have to be properly defined, to capture all possible cases where the control flow is modified by divergence.

- finally, a single coaxiom has to be introduced, stating that the only admissible result for judgments derived with infinite proof trees is divergence (that is, $\infty$ ); this allows filtering out all spurious results different from divergence when the proof tree for the coevaluation judgment is infinite.

We have shown that such an extension is conservative, in the sense that it preserves the inductive semantics of standard rules, hence, the semantics of terminating computations is not affected. Under this conservativity assumption a divergence consistency principle can be conveniently used for proving properties involving divergent computations.

We have studied the approach for two different example languages, the $\lambda$-calculus (Sect. 3), and an imperative FJ-like language (Sect. 4); for the $\lambda$-calculus we have proved the equivalence of our big-step semantics with the standard small-step semantics, and then shown how type soundness can be proved for the simply-typed $\lambda$-calculus; for both properties, the divergence consistency principle can be exploited to simplify the proofs. The same technique followed for the $\lambda$-calculus is adopted in Sect. 5 to prove type soundness of the FJ-like imperative language. For the FJ-like language we did not prove equivalence with the small-step semantics for space limitations, but we expect that the same proof techniques used for the $\lambda$-calculus can be applied also in this case.

To assess the applicability and generality of our approach much work is still needed. We are currently considering to apply coaxioms to other kinds of semantics; in particular, trace semantics seems particularly interesting for investigating whether our approach is suitable for formalizing and proving important safety properties of non terminating programs, as ensuring that a server will never try to use infinite resources. Other approaches based on definitional interpreters [Danielsson 2012], and the adoption of step counters [Amin and Rompf 2017; Ancona 2014; Ernst et al. 2006; Owens et al. 2016] seem to be appealing for proving type soundness properties with big-step semantics, and other results concerning program equivalence; however, it is not clear whether these solutions work well for other kinds of properties. For instance, if a program consists of an infinite loop that allocates new heap space at each step without releasing it, one would like to conclude that it will eventually crash even though a definitional interpreter returns timeout for all possible values of the step counter.

Besides experimenting with other kinds of semantics, also more realistic languages should be considered with additional features, such as exceptions. Then it would be interesting to study whether non-determinism could be addressed to be able to formalize the standard specification of languages where, for instance, no particular evaluation order is fixed in some cases (consider, for instance, computing the arguments in a $\mathrm{C}$ function call).

Finally, to gain greater confidence in the effectiveness of our approach to prove program properties in realistic programming languages, it would be useful to check and mechanize proofs with the support of a theorem prover. 


\section{A PROOF OF PROGRESS LEMMA FOR THE FJ-LIKE LANGUAGE}

In order to prove the progress lemma we need some auxiliary definitions and lemmas provided below. We start introducing a notion of coherence between heaps.

Definition A.1. Given two heaps $\mathcal{H}$ and $\mathcal{H}^{\prime}, \mathcal{H}^{\prime}$ is coherent with $\mathcal{H}$, written $\mathcal{H}^{\prime} \leq \mathcal{H}$, iff $\operatorname{dom}(\mathcal{H}) \subseteq \operatorname{dom}\left(\mathcal{H}^{\prime}\right)$, and for all types $\tau$, and all $\iota \in \operatorname{dom}(\mathcal{H})$, if $\mathcal{H} \vdash \iota: \tau$, then $\mathcal{H}^{\prime} \vdash \iota: \tau$.

The coherence relation $\mathcal{H}^{\prime} \leq \mathcal{H}$ models the fact that $\mathcal{H}^{\prime}$ can be produced by evaluating some well-typed expression starting from $\mathcal{H}$, this will be made more precise in Lemma A.10. Now we state and prove some lemmas useful to prove Lemma A.10 and Lemma 5.4, starting from some properties of coherence relation. First of all we notice that $\leq$ is a preorder. This is useful, in combination with Lemma A.10, to infer coherence between the starting and the resulting heaps in a sequence of computations.

Lemma A.2. The coherence relation $\mathcal{H}^{\prime} \leq \mathcal{H}$ is a pre-order.

Proof. A direct consequence of the fact that set inclusion and logical implication are reflexive and transitive.

The following two lemmas state that agreement between type environment, stack frame and heap, and types of values are preserved by the coherence relation.

Lemma A.3. If $(\Pi ; \mathcal{H}): \Gamma$ and $\mathcal{H}^{\prime} \leq \mathcal{H}$, then $\left(\Pi ; \mathcal{H}^{\prime}\right): \Gamma$.

Proof. A direct consequence of Def. 5.1 and Def. A.1.

Lemma A.4. If $\mathcal{H} \vdash v: \tau, \mathcal{H}^{\prime} \leq \mathcal{H}$, and $\tau \leq \tau^{\prime}$, then $\mathcal{H}^{\prime} \vdash v: \tau^{\prime}$.

Proof. If $v=$ false or $v=$ true, then $\tau=$ bool, and either $\tau^{\prime}=$ bool or $\tau=$ object, or $\tau=\tau^{\prime}=$ object, and for all cases $\mathcal{H}^{\prime} \vdash v: \tau^{\prime}$.

If $v=\imath$, then by rule (v-oвJ), $\iota \in \operatorname{dom}(\mathcal{H})$; since $\mathcal{H}^{\prime} \leq \mathcal{H}$, by Def. A.1, $\mathcal{H}^{\prime} \vdash \iota: \tau$, and by rule (v-oвJ), $\mathcal{H}^{\prime}(\iota)=\operatorname{obj}(c,\{\bar{f} \mapsto \bar{v}\}), \forall i=1 . . n . \mathcal{H}^{\prime} \vdash v_{i}: \tau_{i}, c \leq \tau$, and fields $(c)=\overline{\tau f}$. By transitivity of subtyping $c \leq \tau^{\prime}$, therefore, by rule (v-oвJ), $\mathcal{H}^{\prime} \vdash l: \tau^{\prime}$.

Lemma A.5. $\tau_{1} \leq \vee\left(\tau_{1}, \tau_{2}\right)$ and $\tau_{2} \leq \vee\left(\tau_{1}, \tau_{2}\right)$.

Proof. Straightforward induction on the rules defining $\vee\left(\tau_{1}, \tau_{2}\right)$.

The following two lemmas allow us to infer information about values of functions fields and meth of a class $c^{\prime}$ from those of one of its superclasses $c$. This is particularly useful to get information about the dynamic type of an object starting from its static type.

LemmA A.6. If fields $(c)=\overline{\tau f}^{n}$, fields $\left(c^{\prime}\right)={\overline{\tau^{\prime} f^{\prime}}}^{m}$, and $c^{\prime} \leq c$, then $n \leq m$, and for all $i=1 . . n$, $\tau_{i}=\tau_{i}^{\prime}$, and $f_{i}=f_{i}^{\prime}$.

Proof. Straightforward induction on the rules defining $c^{\prime} \leq c$.

Lemma A.7. Let $\overline{c d}$ be well-typed class declarations. If in $\overline{c d}$ meth $(c, m)=\overline{\tau x}^{n} . e: \tau$ and $c^{\prime} \leq c$, then meth $\left(c^{\prime}, m\right)={\overline{\tau^{\prime} x^{\prime}}}^{n} . e^{\prime}: \tau^{\prime}, \tau^{\prime} m\left({\overline{\tau^{\prime} x^{\prime}}}^{n}\right)\left\{e^{\prime}\right\}$ is declared in a class $c^{\prime \prime}$ s.t. $c^{\prime} \leq c^{\prime \prime} \leq c$, and for all $i=1 . . n \tau_{i} \leq \tau_{i}^{\prime}$ and $\tau^{\prime} \leq \tau$.

Proof. Straightforward induction on the rules defining $c^{\prime} \leq c$.

These last two auxiliary lemmas ensure that creating a new reference in a heap and updating the value of an object's field are operations that generate a new heap coherent with the previous one. 
Lemma A.8. If $\iota \notin \operatorname{dom}(\mathcal{H})$ and for all $f \in \operatorname{dom}(\rho), \rho(f) \in \operatorname{dom}(\mathcal{H}) \cup\{\iota\}$, then $\mathcal{H} \uplus\{\iota \mapsto \operatorname{obj}(c, \rho)\} \leq$ $\mathcal{H}$.

Proof. The prove proceeds by coinduction on the definition of $\mathcal{H} \vdash v: \tau$ and exploits the trivial facts that $\mathcal{H}^{\prime}=\mathcal{H} \uplus\{\iota \mapsto \operatorname{obj}(c, \rho)\}$ iff $\left(\iota \in \operatorname{dom}\left(\mathcal{H}^{\prime}\right)\right.$ and $\left.\mathcal{H}=\mathcal{H}^{\prime} \backslash\{\iota \mapsto o b j(c, \rho)\}\right)$ and that if $\mathcal{H}\left(\iota^{\prime}\right)$ is defined, then $\iota^{\prime} \neq \iota$, and $\mathcal{H}\left(\iota^{\prime}\right)=(\mathcal{H} \uplus\{\iota \mapsto \operatorname{obj}(c, \rho)\})\left(\iota^{\prime}\right)$.

Lemma A.9. If $\mathcal{H}^{\prime}=\mathcal{H} \uplus\left\{\iota \mapsto o b j\left(c, \rho \uplus\left\{f \mapsto v_{f}\right\}\right)\right\}, \tau$ fin fields $(c)$, and $\mathcal{H}^{\prime} \vdash u_{f}: \tau$, then $\mathcal{H} \uplus\left\{\iota \mapsto o b j\left(c, \rho \uplus\left\{f \mapsto u_{f}\right\}\right)\right\} \leq \mathcal{H}^{\prime}$.

Proof. Analogously to the previous lemma, the prove proceeds by coinduction on the definition of $\mathcal{H} \vdash v: \tau$. The case $v \neq \iota$ is straightforward; if $v=\iota$, then the coinduction principle can be applied by virtue of the hypotheses $\tau f$ in fields $(c)$, and $\mathcal{H}^{\prime} \vdash u_{f}: \tau$.

Now we are ready to prove the type preservation lemma.

Lemma A.10 (Type PREservation). If $\Gamma \vdash e: \tau, \Pi ; \mathcal{H} ; e \Rightarrow v ; \Pi^{\prime} ; \mathcal{H}^{\prime}$ and $(\Pi ; \mathcal{H}): \Gamma$ hold, then

(1) $\mathcal{H}^{\prime} \leq \mathcal{H}$

(2) $\mathcal{H}^{\prime} \vdash v: \tau$ and $\left(\Pi^{\prime} ; \mathcal{H}^{\prime}\right): \Gamma$

Proof. We prove both statements by induction on the rules of the big step semantics; this is possible thanks to Theorem 4.1.

(1) For rules (VAR), (TRU), (FAL) there is nothing to prove. For rule (NEw), the thesis follows from the inductive hypothesis, Lemma A.8 and Lemma A.2. For rule (IFT), (IFF), (FLD) and (VAS), the thesis follows from the inductive hypothesis and Lemma A.2. For rule (inv) first note that by rule (T-INv) premises are well typed and $\operatorname{meth}\left(\tau_{0}, m\right)=\bar{\tau}^{n} . e: \tau$ and, since class declarations are well typed, $\Gamma^{\prime} \vdash e: \tau$ holds, with $\Gamma^{\prime}=\left\{\right.$ this : $\left.\tau_{0}, \overline{x: \tau^{n}}\right\}$; furthermore, by hypothesis $\mathcal{H}_{1}\left(v_{0}\right)=\operatorname{obj}(c, \rho)$ with $c \leq \tau_{0}$, meth $(c, m)={\overline{\tau^{\prime} x^{\prime}}}^{n} . e^{\prime}: \tau^{\prime}$ and by Lemma A.7 $\tau^{\prime} \leq \tau$ and $\tau_{i} \leq \tau_{i}^{\prime}$ for all $i=1 . . n$, so using the inductive hypothesis, Lemma A.2, Lemma A.4, Def. 5.1 and rule (v-овJ) $\left(\Pi^{\prime} ; \mathcal{H}_{n+1}\right): \Gamma^{\prime}$ holds ${ }^{7}$, with $\Pi^{\prime}=\left\{\right.$ this $\left.\mapsto v_{0}, \bar{x}^{n} \mapsto \bar{v}^{n}\right\}$. Thus the thesis follows from the inductive hypothesis and Lemma A.2. For rule (FAs), the thesis follows from the inductive hypothesis, Lemma A.9 and Lemma A.2.

(2) For rules (TRU) and (FAL) the thesis is trivial.

For rule (var) the thesis holds since by hypothesis $(\Pi ; \mathcal{H}): \Gamma$, therefore by Def. $5.1, \mathcal{H}$ ト $\Pi(x): \Gamma(x)$.

For rule (NEw) by rule (T-NEw) premises are well typed, so by inductive hypothesis $\mathcal{H}_{i} \vdash v_{i}: \tau_{i}$ holds for all $i=1 . . n$, by Lemma A.8 $\mathcal{H}^{\prime}=\mathcal{H}_{n} \uplus\left\{\iota \mapsto \operatorname{obj}\left(c, \bar{f}^{n} \mapsto \bar{v}^{n}\right)\right\} \leq \mathcal{H}_{n}$ and by Lemma A.2 for all $i=1 . . n, \mathcal{H}^{\prime} \leq \mathcal{H}_{i}$, that implies $\mathcal{H}^{\prime} \vdash v_{i}: \tau_{i}$ by Lemma A.4 and so by rule (v-овJ) $\mathcal{H}^{\prime} \vdash$ l:c.

For rule (IFT) and (IFF) the thesis follows from the inductive hypothesis, Lemma A.5 and point (1).

For rule (FLD) by hypothesis and rule (T-FLD) $\Gamma \vdash e: c$ for some class $c$. By inductive hypothesis $\mathcal{H}_{1} \vdash \iota: c$ and $\mathcal{H}_{1}(\iota)=o b j\left(c^{\prime}, \rho\right)$ with $c^{\prime} \leq c$, by Lemma A.6 $\tau f$ is in fields $\left(c^{\prime}\right)$ and by rule (v-oвJ) $\mathcal{H}_{1} \vdash \rho(f): \tau$.

For rule (INv) as noted previously $\left(\Pi^{\prime} ; \mathcal{H}_{n+1}\right): \Gamma^{\prime}$ where $\Gamma^{\prime}=\left\{\right.$ this $\left.: \tau_{0}, \overline{x: \tau^{n}}\right\}$ and $\Pi^{\prime}=$ $\left\{\right.$ this $\mapsto v_{0},{\overline{x^{\prime}}}^{n} \mapsto \bar{v}^{n}$ \} holds. Therefore by inductive hypothesis for all $i=0 . . n, \mathcal{H}_{i+1} \vdash v_{i}: \tau_{i}$ and $\left(\Pi_{i+1} ; \mathcal{H}_{i+1}\right): \Gamma$ hold and also $\mathcal{H}_{n+2} \vdash v: \tau$ holds. So, since $\mathcal{H}_{n+2} \leq \mathcal{H}_{n+1}$, by point (1), we get $\left(\Pi_{n+1} ; \mathcal{H}_{n+2}\right): \Gamma$ by Lemma A.3.

\footnotetext{
${ }^{7}$ modulo renaming of variables.
} 
For rule (vas) by rule (T-vas) $\Gamma \vdash e: \tau, \Gamma(x)=\tau^{\prime}$ and $\tau \leq \tau^{\prime}$ and by inductive hypothesis $\mathcal{H}_{1} \vdash v: \tau$ and $\left(\Pi^{\prime} ; \mathcal{H}_{1}\right): \Gamma$ where $\Pi^{\prime}=\Pi_{1} \uplus\left\{x \mapsto v^{\prime}\right\}$. So, noting that by rule (v-овJ) we have $\mathcal{H}_{1} \vdash v: \tau^{\prime}$, we get $\left(\Pi^{\prime \prime} ; \mathcal{H}_{1}\right): \Gamma$ where $\Pi^{\prime \prime}=\Pi_{1} \uplus\{x \mapsto v\}$.

For rule (FAs) by inductive hypothesis $\mathcal{H}_{2} \vdash v: \tau$ and $\left(\Pi_{2} ; \mathcal{H}_{2}\right): \Gamma$, therefore the thesis follows from Lemma A.9, Lemma A.4 and Lemma A.3.

Now we are ready to prove Lemma 5.4.

Proof of Lemma 5.4 (Progress lemma). We proceed by case analysis on $e$.

$e=$ true $\vee e=$ false Empty cases since by rule (TRU) and (FAL) boolean values evaluate to themselves.

$e=x$ Empty case since by rule (T-VAR) we have $\Gamma(x)=\tau$ and since $(\Pi ; \mathcal{H}): \Gamma$ by Def. $5.1 x \in$ $\operatorname{dom}(\Pi)$; therefore by rule (var) we get $\Pi ; \mathcal{H} ; x \Rightarrow \Pi(x) ; \Pi ; \mathcal{H}$.

$e=$ new $c(\bar{e})$ By rule (T-New) we have that for all $i=1 . . n \Gamma \vdash e_{i}: \tau_{i}$, fields $(c)=\overline{\tau^{\prime} f}$ and $\tau_{i} \leq \tau_{i}^{\prime}$. Set $\Pi_{0}=\Pi$ and $\mathcal{H}_{0}=\mathcal{H}$, we have two cases:

- If for all $i=1 . . n \Pi_{i-1} ; \mathcal{H}_{i-1} ; e_{i} \Rightarrow v_{i} ; \Pi_{i} ; \mathcal{H}_{i}$ holds, then by rule (NEw) $e$ converges, and this contradicts the hypothesis of the lemma;

- If there exists a first index $i$ s.t. there is no $r_{i}=v_{i} ; \Pi_{i} ; \mathcal{H}_{i}$ s.t. $\Pi_{i-1} ; \mathcal{H}_{i-1} ; e_{i} \Rightarrow r_{i}$ and for all $j=1 . . i-1, \Pi_{j-1} ; \mathcal{H}_{j-1} ; e_{j} \Rightarrow v_{j} ; \Pi_{j} ; \mathcal{H}_{j}$ holds; then by Lemma A.10 we get also that for all $j=1 . . i-1,\left(\Pi_{j} ; \mathcal{H}_{j}\right): \Gamma$ and in particular $\left(\Pi_{i-1} ; \mathcal{H}_{i-1}\right): \Gamma$, so $\Pi_{i-1} ; \mathcal{H}_{i-1} ; e_{i} \in W T^{\infty}$ and we conclude by rule (Div).

$e=e^{\prime} . f$ By rule (T-FLD) we get that $\Gamma \vdash e^{\prime}: c$ and fields $(c)=\overline{\tau f}$ with $f=f_{i}$ and $\tau=\tau_{i}$ for some

$i=1$..n. We have two cases:

- If $\Pi ; \mathcal{H} ; e^{\prime} \Rightarrow v ; \Pi^{\prime} ; \mathcal{H}^{\prime}$ holds for some $r^{\prime}=v ; \Pi^{\prime} ; \mathcal{H}^{\prime}$, then by Lemma A.10 $\mathcal{H}^{\prime} \vdash v: c$ holds, that implies, by rule (v-овJ), $\mathcal{H}^{\prime}(v)=\operatorname{obj}\left(c^{\prime},\left\{\bar{f}^{n} \mapsto \bar{v}^{n}\right\}\right)$ with $c^{\prime} \leq c$ and by Lemma A.6 $f$ is a field of $c^{\prime}$, therefore by rule (FLD) we get that $\Pi ; \mathcal{H} ; e \Rightarrow v_{i} ; \Pi^{\prime} ; \mathcal{H}^{\prime}$ holds, and this contradicts the hypothesis of the lemma;

- If there is no $r$ s.t. $\Pi ; \mathcal{H} ; e^{\prime} \Rightarrow r$, then, $\Pi ; \mathcal{H} ; e^{\prime} \in W T^{\infty}$ and so we conclude by rule (Div). $e=e_{0} \cdot m\left(\bar{e}^{n}\right)$ By rule (T-INv) for all $i=0 . . n, \Gamma \vdash e_{i}: \tau_{i}, \operatorname{meth}\left(\tau_{0}, m\right)={\overline{\tau^{\prime} x}}^{n} \cdot e^{\prime}: \tau$ and for all $i=1 . . n \tau_{i} \leq \tau_{i}^{\prime}$. Set $\Pi_{0}=\Pi$ and $\mathcal{H}_{0}=\mathcal{H}$, and note that if $\Pi_{0} ; \mathcal{H}_{0} ; e_{0} \Rightarrow v_{0} ; \Pi_{1} ; \mathcal{H}_{1}$ holds, then by Lemma A.10 we get $\mathcal{H}_{1} \vdash v_{0}: \tau_{0}$ and by rule ${ }_{(\mathrm{v} \text {-овJ) }} \mathcal{H}_{1}\left(v_{0}\right)=\operatorname{obj}\left(\tau_{0}^{\prime}, \rho\right)$ with $\tau_{0}^{\prime} \leq \tau_{0}$; furthermore by Lemma A.7 we have $\operatorname{meth}\left(\tau_{0}^{\prime}, m\right)={\overline{\tau^{\prime} x}}^{n} \cdot e^{\prime}: \tau^{\prime}$ with $\tau^{\prime} \leq \tau$ and $\tau_{i}^{\prime \prime} \leq \tau_{i}^{\prime}$ for all $i=1 . . n$. We have three cases

- if for all $i=0 . . n \Pi_{i} ; \mathcal{H}_{i} ; e_{i} \Rightarrow v_{i} ; \Pi_{i+1} ; \mathcal{H}_{i+1}$ and $\Pi^{\prime} ; \mathcal{H}_{n+1} ; e^{\prime} \Rightarrow r$ where $\Pi^{\prime}=\{$ this $\mapsto$ $\left.v_{0}, \bar{x}^{n} \mapsto \bar{v}^{n}\right\}$, thanks to the observation above, by rule (inv) we get $\Pi ; \mathcal{H} ; e \Rightarrow r$, and this contradicts the hypothesis of the lemma;

- if for all $i=0 . . n \Pi_{i} ; \mathcal{H}_{i} ; e_{i} \Rightarrow v_{i} ; \Pi_{i+1} ; \mathcal{H}_{i+1}$, but $\Pi^{\prime} ; \mathcal{H}_{n+1} ; e^{\prime} \Rightarrow r$ where $\Pi^{\prime}=\{$ this $\mapsto$ $\left.v_{0}, \bar{x}^{n} \mapsto \bar{v}^{n}\right\}$ does not hold, then $\left(\Pi^{\prime} ; \mathcal{H}_{n+1}\right): \Gamma^{\prime}$ holds where $\Gamma^{\prime}=\left\{\right.$ this : $\left.\tau_{0}^{\prime},{\overline{x: \tau^{\prime}}}^{n}\right\}$, indeed $\operatorname{dom}\left(\Gamma^{\prime}\right) \subseteq \operatorname{dom}\left(\Pi^{\prime}\right)$ by construction and $\mathcal{H}_{n+1} \vdash v_{i}: \tau_{i}^{\prime}$ for all $i=0 . . n$ because, by Lemma A.10, $\mathcal{H}_{n+1} \leq \mathcal{H}_{i}$ and $\mathcal{H}_{i+1} \vdash v_{i}: \tau_{i}$ for all $i=0 . . n$, by Lemma A.4 $\mathcal{H}_{n+1} \vdash v_{i}: \tau_{i}$ for all $i=0 . . n$ and by definition $\mathcal{H}_{n+1} \vdash v_{i}: \tau_{i}^{\prime}$ for all $i=0 . . n$ since $\tau_{i} \leq \tau_{i}^{\prime \prime} \leq \tau_{i}^{\prime}$. Hence by definition $\Pi^{\prime} ; \mathcal{H}_{n+1} ; e^{\prime} \in W T^{\infty}$, and so we conclude by rule (INv);

- if there exists $i=0 . . n$ s.t. $\Pi_{i} ; \mathcal{H}_{i} ; e_{i} \Rightarrow r_{i}$ does not hold for any $r_{i}$, and for all $j=0 . . i-1$, $\Pi_{j} ; \mathcal{H}_{j} ; e_{j} \Rightarrow v_{j} ; \Pi_{j+1} ; \mathcal{H}_{j+1}$ holds, then by Lemma A.10 for all $j=0 . . i-1,\left(\Pi_{j+1} ; \mathcal{H}_{j+1}\right): \Gamma$ and in particular $\left(\Pi_{i} ; \mathcal{H}_{i}\right): \Gamma$. Therefore by definition $\Pi_{i} ; \mathcal{H}_{i} ; e_{i} \in W T^{\infty}$, and so we conclude by rule (Div). 
$e=$ if $\left(e_{0}\right) e_{1}$ else $e_{2}$ By rule (T-IF) we get that $\Gamma \vdash e_{0}:$ bool, $\Gamma \vdash e_{1}: \tau_{1}$ and $\Gamma \vdash e_{2}: \tau_{2}$ with $\tau=\vee\left(\tau_{1}, \tau_{2}\right)$. We have two cases:

- if $\Pi ; \mathcal{H} ; e_{0} \Rightarrow v ; \Pi^{\prime} ; \mathcal{H}^{\prime}$ holds, then by Lemma A.10 $\mathcal{H}^{\prime} \vdash v$ :bool, that implies by rule (vвоо--2) either $v=$ true or $v=$ false. Suppose $v=$ true (the case $v=$ false is symmetric). Now if $\Pi^{\prime} ; \mathcal{H}^{\prime} ; e_{1} \Rightarrow r$ holds by rule (IFT) we conclude $\Pi ; \mathcal{H} ; e \Rightarrow r$, and this contradicts the hypothesis of the lemma. If, on the other hand, there is no $r$ s.t. $\Pi^{\prime} ; \mathcal{H}^{\prime} ; e_{1} \Rightarrow r$, then by Lemma A.10 $\left(\Pi^{\prime} ; \mathcal{H}^{\prime}\right): \Gamma$, hence by definition $\Pi^{\prime} ; \mathcal{H}^{\prime} ; e_{1} \in W T^{\infty}$, therefore we conclude by rule (IFT).

- If there is no $r$ s.t. $\Pi ; \mathcal{H} ; e_{0} \Rightarrow r$ then by definition $\Pi ; \mathcal{H} ; e_{0} \in W T^{\infty}$, therefore we conclude by rule (Div).

$e=x=e^{\prime}$ By rule (T-vas) we get that $\Gamma \vdash e^{\prime}: \tau, \Gamma(x)=\tau^{\prime}$ and $\tau \leq \tau^{\prime}$. We have two cases:

- if $\Pi ; \mathcal{H} ; e^{\prime} \Rightarrow v ; \Pi^{\prime} ; \mathcal{H}^{\prime}$ holds for some $r=v ; \Pi^{\prime} ; \mathcal{H}^{\prime}$, then by Lemma A.10 we get $\left(\Pi^{\prime} ; \mathcal{H}^{\prime}\right): \Gamma$ that implies, by Def. 5.1, $x \in \operatorname{dom}\left(\Pi^{\prime}\right)$; therefore by rule (vas) we conclude that $\Pi ; \mathcal{H} ; e \Rightarrow$ $v ; \Pi^{\prime \prime} ; \mathcal{H}^{\prime}$, and this contradicts the hypothesis of the lemma;

- if there is no $r$ s.t. $\Pi ; \mathcal{H} ; e^{\prime} \Rightarrow r$, then by definition $\Pi ; \mathcal{H} ; e^{\prime} \in W T^{\infty}$, thus we conclude by rule (Div).

$e=e_{1} \cdot f=e_{2}$ By rule (T-FAs) we get $\Gamma \vdash e_{1}: c$, fields $(c)=\overline{\tau f}^{n}$ with $f=f_{i}, \Gamma \vdash e_{2}: \tau$ and $\tau \leq \tau_{i}$.

Set $\Pi_{0}=\Pi$ and $\mathcal{H}_{0}=\mathcal{H}$, we have two cases:

- If for all $i=1,2, \Pi_{i-1} ; \mathcal{H}_{i-1} ; e_{i} \Rightarrow v_{i} ; \Pi_{i} ; \mathcal{H}_{i}$ holds, then by Lemma A.10 we have $\mathcal{H}_{1} \vdash v_{1}: c$, that implies, by rule (v-овJ), that $\mathcal{H}_{1}\left(v_{1}\right)=o b j\left(c^{\prime}, \rho\right)$ with $c^{\prime} \leq c$ and by Lemma A.6 $\tau_{i} f_{i}$ appears in fields $\left(c^{\prime}\right)$; therefore by rule (FAs) we get $\Pi ; \mathcal{H} ; e \Rightarrow v_{2} ; \Pi_{2} ; \mathcal{H}_{2}$, and this contradicts the hypothesis of the lemma;

- If exists $i=1,2$ s.t. there is no $r_{i}$ s.t. $\Pi_{i-1} ; \mathcal{H}_{i-1} ; e_{i} \Rightarrow r_{i}$ and for all $j=1 . . i-1$ we have $\Pi_{j-1} ; \mathcal{H}_{j-1} ; e_{j} \Rightarrow r_{j}$, by Lemma A.10 we have that $\left(\Pi_{i-1} ; \mathcal{H}_{i-1}\right): \Gamma$. Therefore by definition $\Pi_{i-1} ; \mathcal{H}_{i-1} ; e_{i} \in W T^{\infty}$, hence we conclude by rule (Div).

\section{ACKNOWLEDGMENTS}

We are grateful to the anonymous reviewers for their thorough comments and useful improvement suggestions.

\section{REFERENCES}

Peter Aczel. 1977. An Introduction to Inductive Definitions. In Handbook of Mathematical logic, Jon Barwise (Ed.). North Holland, 739-782.

Mads Sig Ager. 2004. From Natural Semantics to Abstract Machines. In Logic Based Program Synthesis and Transformation LOPSTR 2004, Revised Selected Papers (Lecture Notes in Computer Science), Sandro Etalle (Ed.), Vol. 3573. Springer, 245-261. DOI : http://dx.doi.org/10.1007/11506676_16

Nada Amin and Tiark Rompf. 2017. Type Soundness Proofs with Definitional Interpreters. In ACM Symp. on Principles of Programming Languages 2017, Giuseppe Castagna and Andrew D. Gordon (Eds.). ACM Press, 666-679. DOI : http: //dx.doi.org/10.1145/3009837

Davide Ancona. 2011. Coinductive Big-Step Operational Semantics for Type Soundness of Java-like Languages. In FTf PP'11 - Formal Techniques for fava-like Programs, Stephen N. Freund (Ed.). ACM Press, 5:1-5:6. DOI : http://dx.doi.org/10.1145/ 2076674.2076679

Davide Ancona. 2012. Soundness of Object-Oriented Languages with Coinductive Big-Step Semantics. In ECOOP'12 Object-Oriented Programming (Lecture Notes in Computer Science), James Noble (Ed.), Vol. 7313. Springer, 459-483. DOI : http://dx.doi.org/10.1007/978-3-642-31057-7_21

Davide Ancona. 2014. How to Prove Type Soundness of Java-like Languages without Forgoing Big-Step Semantics. In FTffP'14 - Formal Techniques for fava-like Programs, David J. Pearce (Ed.). ACM Press, 1:1-1:6. DOI: http://dx.doi.org/10. $1145 / 2635631.2635846$

Davide Ancona and Andrea Corradi. 2014. Sound and Complete Subtyping between Coinductive Types for Object-Oriented Languages. In ECOOP'14 - Object-Oriented Programming (Lecture Notes in Computer Science), Richard Jones (Ed.), Vol. 8586. Springer, 282-307. DOI : http://dx.doi.org/10.1007/978-3-662-44202-9_12 
Davide Ancona, Francesco Dagnino, and Elena Zucca. 2017. Generalizing Inference Systems by Coaxioms. In ESOP 2017 European Symposium on Programming (Lecture Notes in Computer Science), Hongseok Yang (Ed.), Vol. 10201. Springer, 29-55. DOI : http://dx.doi.org/10.1007/978-3-662-54434-1_2

Arthur Charguéraud. 2013. Pretty-Big-Step Semantics. In ESOP 2013 - European Symposium on Programming (Lecture Notes in Computer Science), Matthias Felleisen and Philippa Gardner (Eds.), Vol. 7792. Springer, 41-60. DOI: http: //dx.doi.org/10.1007/978-3-642-37036-6_3

Patrick Cousot and Radhia Cousot. 1992. Inductive Definitions, Semantics and Abstract Interpretations. In ACM Symp. on Principles of Programming Languages 1992, Ravi Sethi (Ed.). ACM Press, 83-94. DOI : http://dx.doi.org/10.1145/143165. 143184

Patrick Cousot and Radhia Cousot. 2009. Bi-inductive structural semantics. Information and Computation 207, 2 (2009), 258-283. DOI : http://dx.doi.org/10.1016/j.ic.2008.03.025

Nils Anders Danielsson. 2012. Operational semantics using the partiality monad. In Intl. Conf. on Functional Programming 2012, Peter Thiemann and Robby Bruce Findler (Eds.). ACM Press, 127-138. DOI : http://dx.doi.org/10.1145/2364527.2364546

Sophia Drossopoulou, Susan Eisenbach, and Sarfraz Khurshid. 1999. Is the Java Type System Sound? Theory and Practice of Object Systems 5, 1 (1999), 3-24.

Erik Ernst, Klaus Ostermann, and William R. Cook. 2006. A virtual class calculus. In ACM Symp. on Principles of Programming Languages 2006, J. Gregory Morrisett and Simon L. Peyton Jones (Eds.). ACM Press, 270-282. DOI : http://dx.doi.org/10. $1145 / 1111037.1111062$

Atsushi Igarashi, Benjamin C. Pierce, and Philip Wadler. 2001. Featherweight Java: a minimal core calculus for Java and GJ. ACM Transactions on Programming Languages and Systems 23, 3 (2001), 396-450. DOI : http://dx.doi.org/10.1145/503502. 503505

Jaroslaw D. M. Kusmierek and Viviana Bono. 2010. Big-step Operational Semantics Revisited. Fundamenta Informaticae 103, 1-4 (2010), 137-172. DOI : http://dx.doi.org/10.3233/FI-2010-323

Xavier Leroy and Hervé Grall. 2009. Coinductive big-step operational semantics. Information and Computation 207, 2 (2009), 284-304. DOI : http://dx.doi.org/10.1016/j.ic.2007.12.004

Robin Milner. 1978. A Theory of Type Polymorphism in Programming. J. Comput. Syst. Sci. 17, 3 (1978), 348-375. DOI: http://dx.doi.org/10.1016/0022-0000(78)90014-4

Robin Milner and Mads Tofte. 1991. Co-Induction in Relational Semantics. Theoretical Computer Science 87, 1 (1991), $209-220$. DOI : http://dx.doi.org/10.1016/0304-3975(91)90033-X

Keiko Nakata and Tarmo Uustalu. 2009. Trace-Based Coinductive Operational Semantics for While. In Theorem Proving in Higher Order Logics - TPHOLs 2009 (Lecture Notes in Computer Science), Stefan Berghofer, Tobias Nipkow, Christian Urban, and Makarius Wenzel (Eds.), Vol. 5674. Springer, 375-390. DOI : http://dx.doi.org/10.1007/978-3-642-03359-9_26

Keiko Nakata and Tarmo Uustalu. 2010a. A Hoare Logic for the Coinductive Trace-Based Big-Step Semantics of While. In ESOP 2010 - European Symposium on Programming (Lecture Notes in Computer Science), Andrew D. Gordon (Ed.), Vol. 6012. Springer, 488-506. DOI : http://dx.doi.org/10.1007/978-3-642-11957-6_26

Keiko Nakata and Tarmo Uustalu. 2010b. Resumptions, Weak Bisimilarity and Big-Step Semantics for While with Interactive I/O: An Exercise in Mixed Induction-Coinduction. In SOS'10 - Structural Operational Semantics (Electronic Proceedings in Theoretical Computer Science), Luca Aceto and Pawel Sobocinski (Eds.), Vol. 32. 57-75. DOI: http://dx.doi.org/10.4204/ EPTCS.32.5

Johan Östlund and Tobias Wrigstad. 2010. Welterweight Java. In Objects, Models, Components, Patterns, TOOLS 2010 (Lecture Notes in Computer Science), Jan Vitek (Ed.), Vol. 6141. Springer, 97-116. DOI : http://dx.doi.org/10.1007/978-3-642-13953-6_ 6

Scott Owens, Magnus O. Myreen, Ramana Kumar, and Yong Kiam Tan. 2016. Functional Big-Step Semantics. In ESOP 2016 European Symposium on Programming (Lecture Notes in Computer Science), Peter Thiemann (Ed.), Vol. 9632. Springer, 589-615. DOI : http://dx.doi.org/10.1007/978-3-662-49498-1_23

Andrei Stefanescu, Daejun Park, Shijiao Yuwen, Yilong Li, and Grigore Rosu. 2016. Semantics-based program verifiers for all languages. In ACM SIGPLAN Conference on Object-Oriented Programming, Systems, Languages and Applications (OOPSLA 2016), Eelco Visser and Yannis Smaragdakis (Eds.). ACM Press, 74-91. DOI : http://dx.doi.org/10.1145/2983990.2984027

Allen Stoughton. 1997. An Operational Semantics Framework Supporting the Incremental Construction of Derivation Trees. Electronic Notes in Theoretical Computer Science 10 (1997), 122-133. DOI : http://dx.doi.org/10.1016/S1571-0661(05)80693-0

Andrew K. Wright and Matthias Felleisen. 1994. A Syntactic Approach to Type Soundness. Information and Computation 115, 1 (1994), 38-94. DOI : http://dx.doi.org/10.1006/inco.1994.1093 